ARTICLE

DOI: $10.1038 / s 41467-018-07142-9$

\title{
Hedgehog stimulates hair follicle neogenesis by creating inductive dermis during murine skin wound healing
}

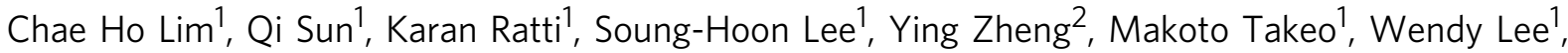

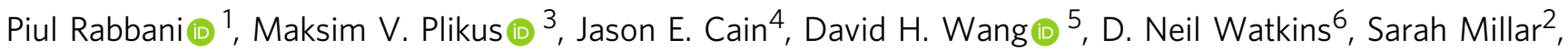 \\ M. Mark Taketo ${ }^{7}$, Peggy Myung (i) ${ }^{8}$, George Cotsarelis ${ }^{2} \&$ Mayumi Ito $^{1}$
}

Mammalian wounds typically heal by fibrotic repair without hair follicle (HF) regeneration. Fibrosis and regeneration are currently considered the opposite end of wound healing. This study sought to determine if scar could be remodeled to promote healing with HF regeneration. Here, we identify that activation of the Sonic hedgehog (Shh) pathway reinstalls a regenerative dermal niche, called dermal papilla, which is required and sufficient for $\mathrm{HF}$ neogenesis (HFN). Epidermal Shh overexpression or constitutive Smoothened dermal activation results in extensive HFN in wounds that otherwise end in scarring. While long-term Wht activation is associated with fibrosis, Shh signal activation in Wnt active cells promotes the dermal papilla fate in scarring wounds. These studies demonstrate that mechanisms of scarring and regeneration are not distant from one another and that wound repair can be redirected to promote regeneration following injury by modifying a key dermal signal.

\footnotetext{
${ }^{1}$ The Ronald O. Perelman Department of Dermatology and Department of Cell Biology, New York University School of Medicine, New York, NY 10016, USA. 2 Department of Dermatology and Cell and Developmental Biology, Perelman School of Medicine, University of Pennsylvania, Philadelphia, PA 19104, USA. ${ }^{3}$ Department of Developmental and Cell Biology, Irvine, Sue and Bill Gross Stem Cell Center, University of California, Irvine, CA 92617, USA. ${ }^{4}$ Centre for Cancer Research, Hudson Institute for Medical Research and Department of Molecular and Translational Science, Faculty of Medicine, Nursing and Health Sciences, Monash University, Clayton, VIC 3816, Australia. ${ }^{5}$ Division of Hematology and Oncology, Department of Internal Medicine, Harold C. Simmons Comprehensive Cancer Center, Esophageal Diseases Center, Medical Service, VA North Texas Health Care System, University of Texas Southwestern Medical Center, 5323 Harry Hines Boulevard, Dallas, TX 75390, USA. ${ }^{6}$ The Kinghorn Cancer Centre, Garvan Institute of Medical Research, Darlinghurst, NSW 2010, Australia. ${ }^{7}$ Division of Experimental Therapeutics, Graduate School of Medicine, Kyoto University, Kyoto 606-8501, Japan. ${ }^{8}$ Departments of Dermatology and Pathology, School of Medicine, Yale University, New Haven, CT 06520, USA. Correspondence and requests for materials should be addressed to M.I. (email: mayumi.ito@nyumc.org)
} 
T he hair follicle (HF) is a complex mini-organ that is formed during embryonic development through communication between follicular epithelial cells and underlying dermal papilla cells $(\mathrm{DP})^{1,2}$. DP cells constitute the dermal niche that instructs hair follicle epithelial cell fate and differentiation ${ }^{3,4}$. After embryonic follicular development, interactions between HF resident stem cells (HFSCs) and the underlying DP continue to regulate the cyclical regeneration of hair shafts in existing HFs throughout the life of the organism ${ }^{5-7}$. However, the de novo generation of HFs is rare in adult animals. Failure to regenerate HFs that are lost from injury or disease represents a major challenge in cutaneous regenerative medicine ${ }^{8,9}$ and can be largely traced to the resistance of adult mammals to resurrect complex embryonic epithelial-mesenchymal interactions that govern organogenesis.

Most adult mammalian tissues do not undergo regenerative healing, a process that recreates a functional tissue nearly indistinguishable from its original form ${ }^{10}$. However, healing of large skin excisions in mice has been shown to recapitulate aspects of true tissue regeneration in the formation of new HFs (HF neogenesis, HFN) thus providing a rare example of mammalian epimorphic regeneration ${ }^{11-13}$. In contrast, small skin excisions in mice result in the formation of scar tissue, or fibrosis, which is composed largely of extracellular matrix components like collagens and fibronectin and devoid of new accessory structures like $\mathrm{HFs}^{11}$ (Supplementary Fig. 1a-g). Both large and small human skin wounds incurred by trauma or dystrophy almost always undergo fibrotic scarring.

As yet, the mediators that determine the balance between scarforming and regenerative skin wound healing are unknown. The relationship between scarring and regeneration is currently elusive. It is unknown whether scarring precludes regeneration, whether scarring cells intrinsically lack the competence for regeneration or whether regenerative cues are simply absent. In this study, we reveal that Shh signaling plays a major role in converting wound fibroblasts from scar promoting, to those that stimulate HFN. Our study suggests that the absence of regenerative cues impacts the inability of hair follicle morphogenesis after injuring the skin.

\section{Results}

Shh signaling is essential for wound-induced HF neogenesis. In analyzing the disparate healing responses in large and small wounds in adult mice, we found that Glil expression, a readout of Hh pathway activation, was localized to the center of large wounds, corresponding to regions of hair placode/germ formation. In striking contrast, it was absent from small wounds (Fig. 1a and Supplementary Fig. 1h). The Gli1 signal in large wounds localized to both DP and epithelial hair germ cells, recapitulating the pattern observed in embryonic HF development $^{14}$. Moreover, Shh, a major ligand of the pathway, was upregulated at the site of HFN in the epithelial compartment in large wounds but absent from either epidermal or dermal compartment in small wounds (Fig. 1b, c).

Shh expression during hair follicle morphogenesis is conserved between mice and humans ${ }^{15}$ and vital for hair follicle development and hair cycle ${ }^{14,16-22}$. We asked if the absence of $\mathrm{Hh}$ pathway activation in mouse small wounds might explain their failure to undergo regenerative wound healing during wound repair.

First, to understand the importance of epithelial Shh expression in large wounds, Shh was genetically deleted from epidermal cells in healed large wounds of K14-CreER; Shh flfl mice upon tamoxifen (TAM) induction from post wound (PW)3d to PW21d. This resulted in a loss of DP and hair germ formation compared to control mice (Fig. 1d-f). These results showed that epithelial Shh ligands are essential for DP formation and HFN. Additionally, deletion of Smo, an essential component of Shh pathway activation, in underlying wound dermal cells in TAMtreated Pdgfra-CreER; Smo fl/fl mice also resulted in inhibition of DP formation and associated HFN events (Fig. 1g-j). Thus, activation of the Shh signaling pathway in the wound dermis plays a vital role in promoting DP formation.

Epithelial Shh leads to HF neogenesis in wounds. To determine if Shh activation could induce HFN in scar-forming wounds, we overexpressed Shh in epithelial cells in K14-CreER; LSL-Shh or K14-CreER; LSL-Shh; Gli1-LacZ mice and examined HFN in small wounds ${ }^{23}$. To induce Shh overexpression, TAM was injected into control and K14-CreER; LSL-Shh or K14-CreER; LSL-Shh; Gli1-LacZ mice from PW1d to indicated time points in Fig. 2. This treatment resulted in extensive HFN in wounds compared to control wounds (Fig. 2a, b). Epidermal Shh overexpression resulted in Gli1 activation in both the epidermis and dermis, corresponding to the areas of HFN. Shh-driven hair germs expressed Lef1 and K17 and exhibited normal hair follicle morphogenesis (Supplementary Fig. 2a-c, f-i). $\mathrm{AP}^{+}$DPs were associated with overlying $\mathrm{K}^{+}{ }^{+}$epithelial buds as typically observed in HFN (Supplementary Fig. 2d) ${ }^{11}$. Eventually, many of these hair follicles $(52 \pm 16 \%$, mean \pm s.d.) grew downward to form mature hair follicles with hair shafts, an event rarely observed in control small wounds (Supplementary Fig. 2e). New DP expressed Lef1 and Noggin as well as AP, further demonstrating their DP identity (Supplementary Fig. 2ff ${ }^{24,25}$. We rarely found aberrant basaloid growths resembling superficial basal-cell carcinomas (BCCs) in these wounds.

Previous studies noted that HFN in large wounds in WT mice was limited to the central area of the wounds ${ }^{11}$. Given the ability of Shh to induce ectopic HFN in small wounds, we asked if exogenous Shh might overcome the inability of new hair follicles to form outside this central region in large wounds. In large wounds from TAM-treated K14-CreER; LSL-Shh mice, we observed extensive DP formation covering the entire wound area compared with TAM-treated controls. (Fig. 2c-e). These results verify the potency of Shh activation to overcome regional inhibition of HFN.

To characterize changes in gene expression following Shh overexpression, TAM was administered into control and K14CreER; LSL-Shh mice from PW1d to PW11d and wound cells isolated for RNA-seq analyses. Epidermal and dermal cells from TAM-treated control and K14-CreER; LSL-Shh mice were compared by RNA-seq analyses (Fig. 2f, g and Table 1 and Supplementary Fig. 3). Gene ontology (GO) analyses showed that processes and signatures involved in embryonic HF morphogenesis, including cell proliferation, cell adhesion, and Hh signaling ${ }^{1}$, were enriched by Shh overexpression (Supplementary Fig. 3a, c). There was no significant difference in expression of Fgf9, which is known to promote hair follicle neogenesis in a large wound model, either in the epidermis (FDR:0.64) or dermis (FDR:0.97) of K14-CreER; LSL-Shh mice compared to controls. Most notably, there was upregulation of the Shh signaling pathway in both the epithelial cells and dermal cells. We also noted upregulation of DP signature genes $B m p 7$, $E n p p 2$, lamc3, and Trps1 in the wound dermis and hair placode signature genes, including Trp73, Vwa2, Samd5, Cxcl14, Nedd9, and Tnfaip 3 in the wound epidermis of Shh overexpressed mice (Supplementary Fig. 3b, d) ${ }^{26}$. These data were confirmed by qPCR analyses (Supplementary Fig. 3e, f). These results suggest that epidermal Shh overexpression can induce key embryonic signatures of HF morphogenesis. 
a

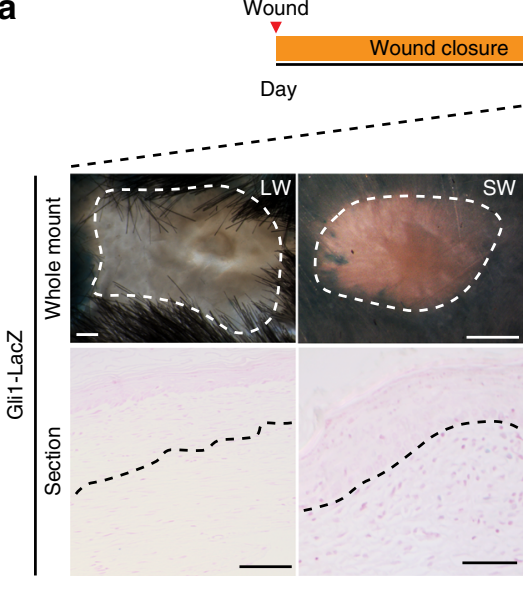

C
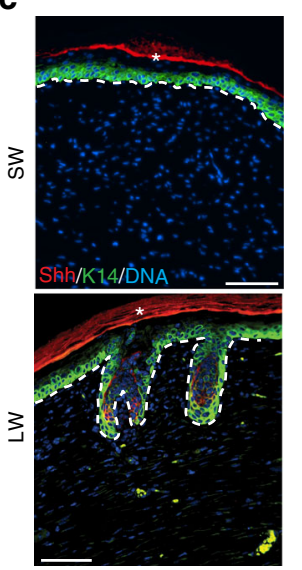

d
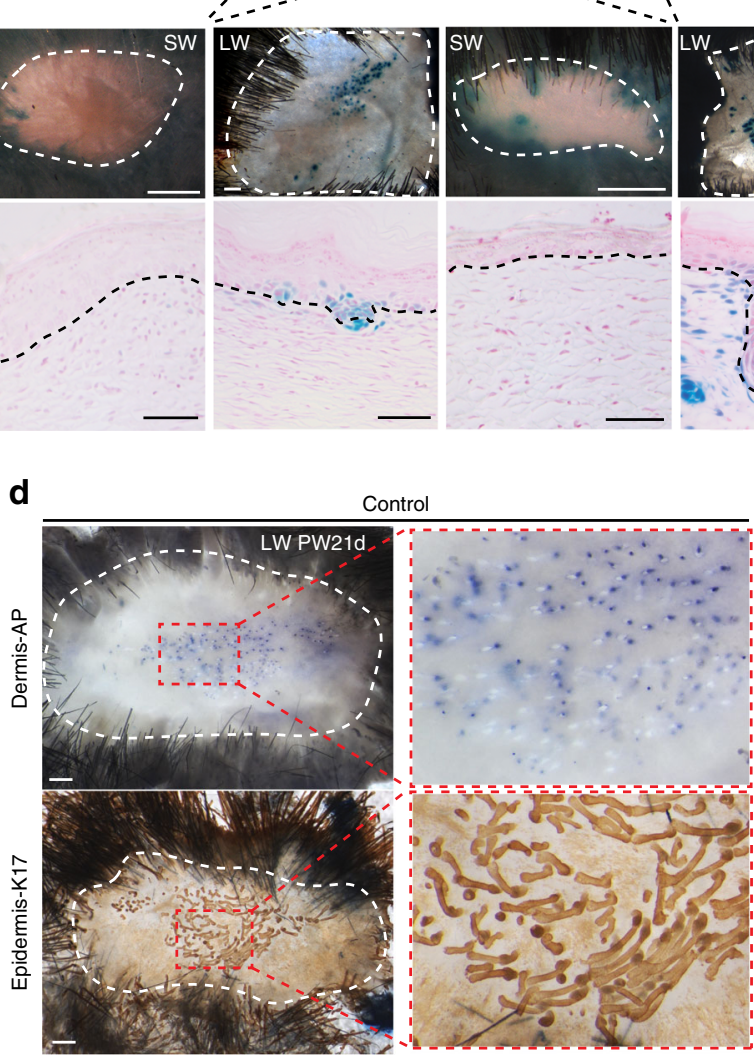
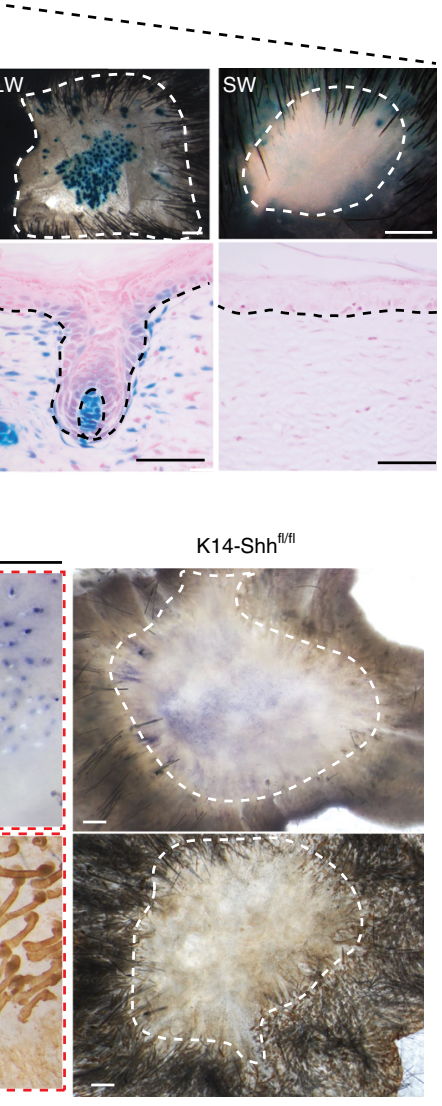

g
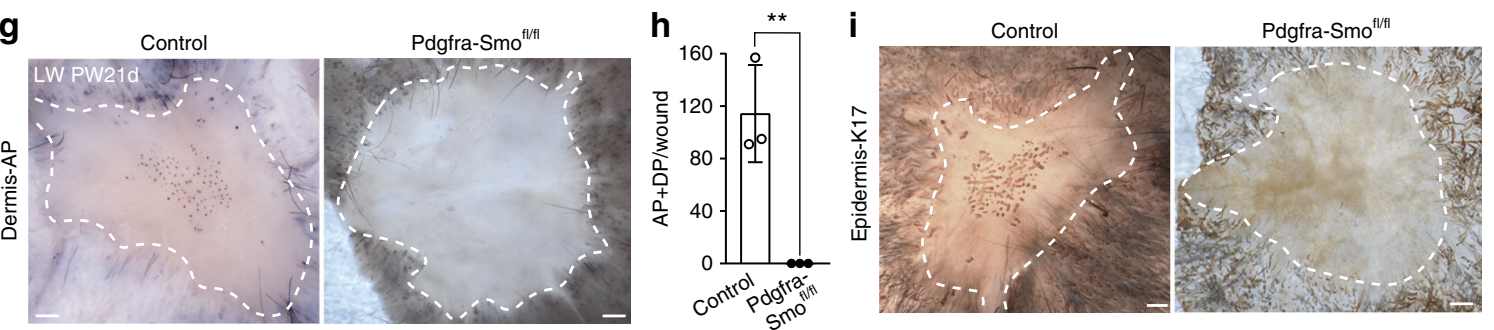
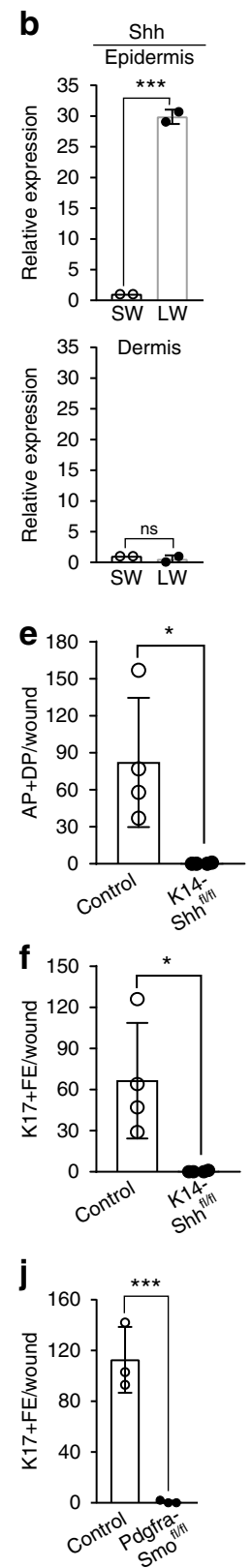

Fig. 1 Shh signaling is necessary for HFN following injury. a X-gal staining of large wound (LW) and small wound (SW) from of GliT-LacZ mice at indicated time ( $n=2-4 \mathrm{~W}$ (2-5 M) per condition). $\mathbf{b}$ qRT-PCR for Shh expression in SW and LW of wild-type mice at 7 days after complete re-epithelialization ( $n=$ 2-6 W (2 M) per condition). c Immunohistochemistry of Shh on SW and LW of wild-type mice at 7 days after complete re-epithelialization. ${ }^{\star}$ Indicates nonspecific signals. d-f K14-CreER; Shh fl/fl (K14- Shh fl/fl) and littermate control mice were subjected to large wound (LW) and treated with TAM from PW3d until tissue harvest at PW21d ( $n=11-12 \mathrm{~W}$ (11-12 M) per condition). Whole-mount HFN assay (d) and quantifications (e, f). g-j Pdgfra-CreER; Smo fl/fl $(P d g f r a-S m o f l / f l)$ and littermate controls were subjected to large wound (LW) and were treated with TAM from 3 or 4 weeks before wounding until tissue harvest at PW21d ( $n=3-5 \mathrm{~W}(3-5 \mathrm{M})$ per condition). Whole-mount HFN assay $(\mathbf{g}, \mathbf{i})$ and quantifications $(\mathbf{h}, \mathbf{j})$. $n$ : number of wounds $(\mathrm{W})$ or mice (M), Data are represented as mean \pm s.d., ${ }^{\star} p<0.05 ;{ }^{\star \star} p<0.01 ;{ }^{\star \star \star} p<0.001$; Student's $t$-test, Dashed white circle: wound boundary, dashed line: epidermis-dermis border, DP dermal papilla, AP alkaline phosphatase, FE follicular epithelium, PW post-wound, Scale bars represent $500 \mu \mathrm{m}$ (a (whole mount), d, $\mathbf{g}, \mathbf{i}), 50 \mu \mathrm{m}$ (a (section), c)

Epithelial Shh regenerates HFs without altering neighboring collagen. Although the increased collagen I deposition by adult dermal fibroblasts vs. fetal fibroblasts during wound healing is well-established, whether the low level of collagen I is essential for embryonic/neonatal non-scarring healing is currently unknown. Our RNA-seq suggests that epithelial Shh overexpression did not significantly change the overall extracellular matrix composition of the wound dermis towards an embryonic state. The increased ratio of type III versus type I collagen is a key characteristic of fetal scarless wound healing ${ }^{27-30}$, and expressions of the genes encoding these collagens were unchanged (FDR: 0.99 for Colla1, Col1a2, and Col3a1) (Table 1). This was verified by biochemical measurement for the content of hydroxyproline, a major component of collagen (Fig. 2h). In addition, transmission electron microscopy (TEM) analysis of the wound area showed no change in collagen fiber diameter due to Shh overexpression, which is directly proportional to the tensile strength ${ }^{31}$ (Fig. 2i). Consistently, no significant differences were noted in the histological 

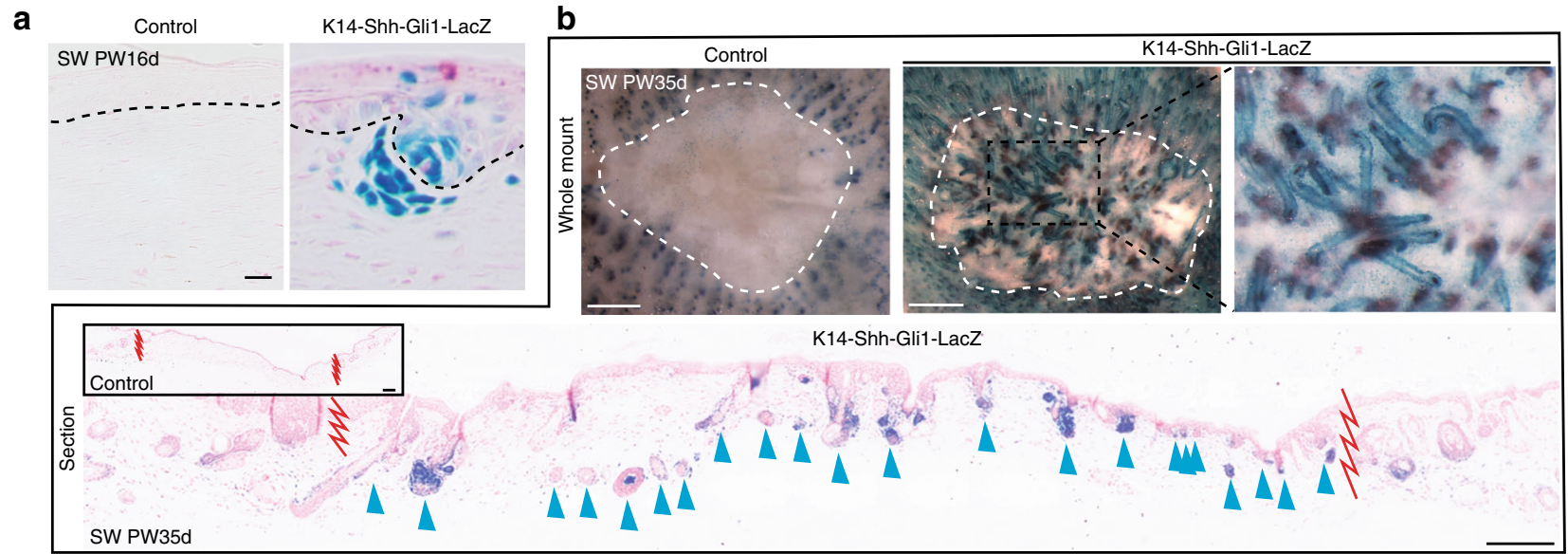

K14-Shh-Gli1-LacZ
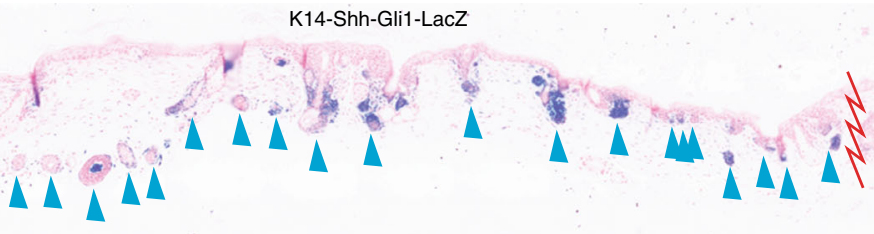

C
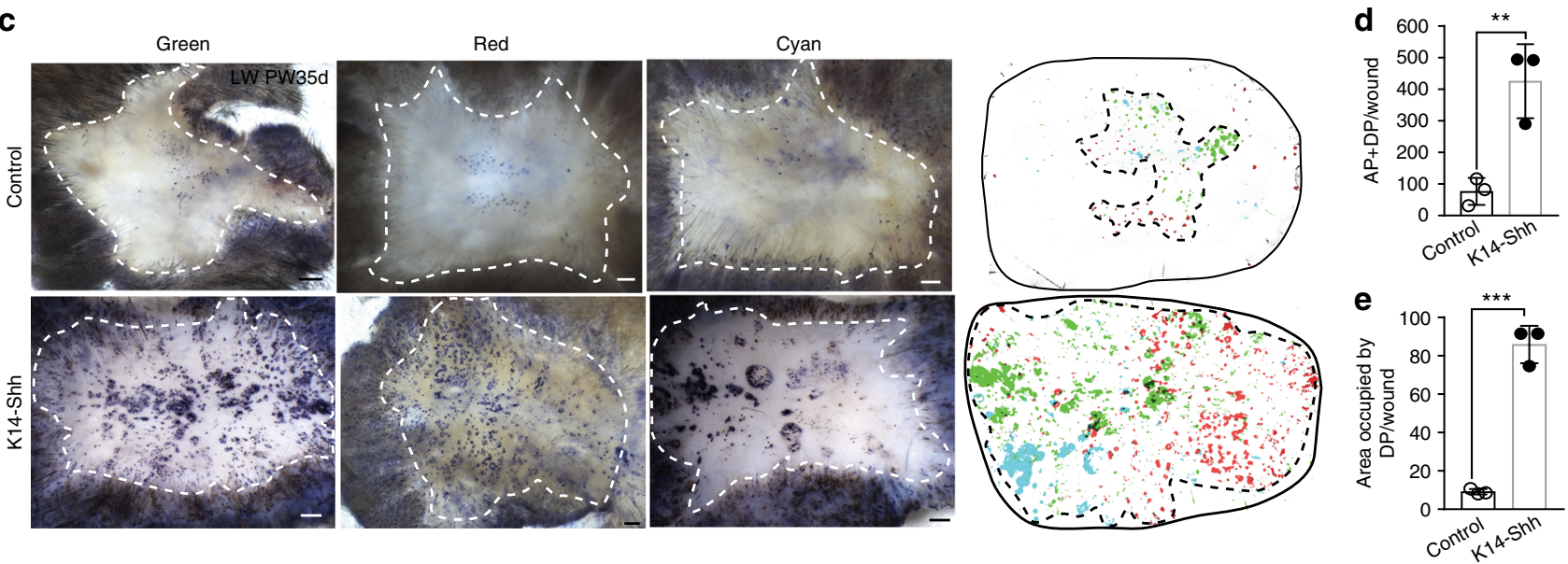

f

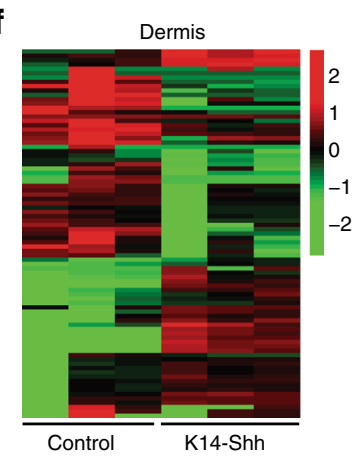

g Epidermis

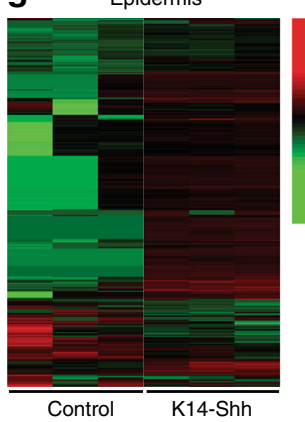

Masson's trichrome

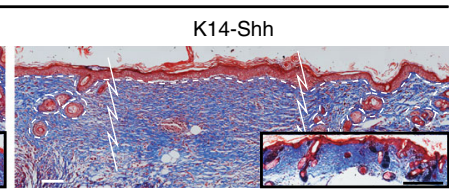

h

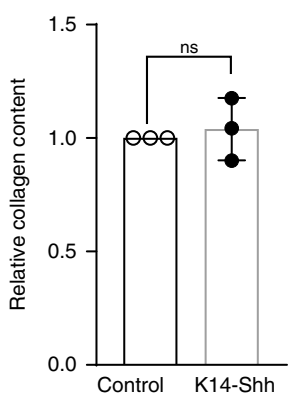

k i
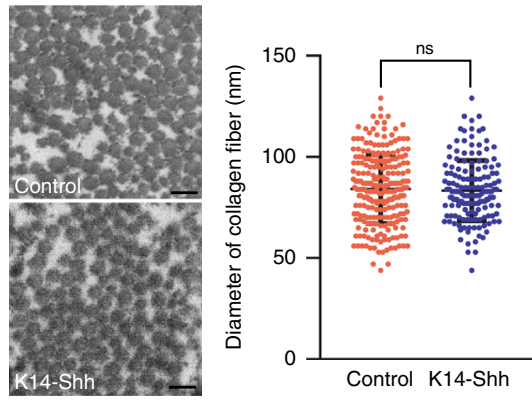

icrosirius red

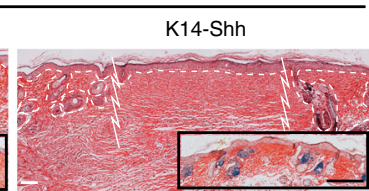

assessments for collagen staining (i.e., Masson trichrome and Picrosirius red staining) before and after the formation of neogenic hair follicles (Fig. 2j, k). We found that the epithelial Shh expression and the reduction of type I collagen in the neighboring wound scar does not play a significant role in Shh-driven HF neogenesis. The data suggests that DP and hair follicles were forming within the scar of the wounds.

Dermal Hh activation induces HF neogenesis in scarring wounds. To ask if direct Shh pathway activation of myofibroblasts in the small wound dermis would also promote DP formation and HFN, we induced expression of the activated form of Smo under the control of the $S M 22 \alpha$ promoter, known to be specifically active only in dermal myofibroblasts (SM22-rtTA; tetO-Cre; R26-SmoM2) (Fig. 3a $)^{32-34}$. In the transgenic mice, constitutively active Smo expression is dependent on doxycycline (dox) administration. Expression of the activated form of Smo in myofibroblasts during wounding (dox treatment from PW1d to PW30d) or following re-epithelialization (dox treatment from PW10 $\pm 2 \mathrm{~d}$ to PW46d) resulted in DP formation within small wounds of SM22-SmoM2 mice compared to control mice (Fig. 3b, c 
Fig. 2 Epidermal Shh is capable of regenerating HF in wounds without alteration of collagen. a, b K14-CreER; LSL-Shh; GliT-LacZ (K14-Shh-Gli7-LacZ) and littermate controls with GliT-LacZ were subjected to SW and treated with TAM from PW1d until tissue harvest at indicated time ( $n=12 \mathrm{~W}$ ( $4 \mathrm{M}$ ) per condition). X-gal staining was analyzed at PW16d (a), and PW35d (b). Arrowheads show regenerated HFs (b). c-e Distribution of regenerated DP was analyzed from three representative LWs of K14-CreER; LSL-Shh (K14-Shh) and littermate controls treated with TAM from PW3d until tissue harvesting at PW35d ( $n=9 \mathrm{~W}(9 \mathrm{M})$ per condition). AP + DP in each picture were converted into dots with three different colors (red, green, and cyan) and merged into one picture (c, right). The original images and the corresponding colors represented in three columns on the left (c). Quantifications of $A P^{+} D^{2}$ per wound (d) and area occupied by DP (e). "Area occupied by DP" was defined by drawing a line that connects the outermost regenerated DP in wound. f-k K14CreER; LSL-Shh (K14-Shh) and littermate controls were subjected to SW and treated with TAM from PW1d until tissue harvest at indicated time. RNA-seq analyses at PW11d showing heatmap of differentially expressed genes for K14-Shh and control mice (f, $\mathbf{g})$. Red and green correspond to high and low expression levels, respectively $(n=12 \mathrm{~W}(4 \mathrm{M})$ per condition). Hydroxyproline assay to measure collagen contents at PW11d ( $n=12 \mathrm{~W}(4 \mathrm{M})$ per condition) (h). Diameter of collagen fiber at PW30d (TEM images on the left, $n=2 \mathrm{~W}(2 \mathrm{M})$ per condition) (i). Detection of collagen fiber by Masson's trichrome staining (j) and Picrosirius red staining (k) at PW11d and PW35d (insets). n: number of wounds (W) or mice (M), Data are represented as mean \pm s.d., ${ }^{\star \star} p<0.01$; ${ }^{\star \star \star} p<0.001$; ns: non-significant; Student's $t$-test, Zigzag line and dashed white circle: wound boundary, Dashed line: epidermis-dermis border, SW small wound, LW large wound, PW post-wound, DP dermal papilla, AP alkaline phosphatase, FE follicular epithelium. Scale bars represent 500 $\mu \mathrm{m}$ (b (whole mount), $\mathbf{c}), 50 \mu \mathrm{m}$ (b (section), j, k), $10 \mu \mathrm{m}$ (a), $100 \mathrm{~nm}$ (i)

\begin{tabular}{|c|c|c|c|c|}
\hline & & Genes & Fold change & FDR \\
\hline \multirow[t]{7}{*}{ Dermis } & \multirow[t]{4}{*}{ Hh pathway } & Shh & 2.41 & 0.64 \\
\hline & & Gli1 & 3.20 & 0.16 \\
\hline & & Gli2 & 4.18 & 0.06 \\
\hline & & Ptch1 & 3.96 & 0.03 \\
\hline & \multirow[t]{3}{*}{ Collagen } & Colla1 & 0.95 & 0.99 \\
\hline & & Colla2 & 1.00 & 0.99 \\
\hline & & Col3a1 & 0.94 & 0.99 \\
\hline \multirow[t]{7}{*}{ Epidermis } & \multirow[t]{4}{*}{ Hh pathway } & Shh & 51.83 & 2.01E-04 \\
\hline & & Gli1 & 4.21 & 1.33E-01 \\
\hline & & Gli2 & 7.27 & 8.65E-07 \\
\hline & & Ptch1 & 3.74 & $3.08 \mathrm{E}-04$ \\
\hline & \multirow[t]{3}{*}{ Collagen } & Colla1 & 0.56 & 0.56 \\
\hline & & Colla2 & 0.82 & 0.87 \\
\hline & & Col3a1 & 0.86 & 0.91 \\
\hline
\end{tabular}

and Supplementary Fig. 4a, b). Constitutive $\mathrm{Hh}$ activation in wound myofibroblasts from SM22-SmoM2 mice also resulted in striking changes in wound epithelial cells. Those epithelial cells directly above Smo-active DP expressed K17 and Shh, wellestablished hair germ markers (Fig. 3d, e, i and Supplementary Fig. 4a, c, 6a). Additionally, these hair germ cells displayed nuclear $\beta$-catenin and Lef1 expression indicating active Wnt signaling, a known signature of hair follicle development and growth (Fig. 3h, j-m) $)^{11,35}$. A significant number of new hair follicles were also observed with differentiated hair shafts $(28$ $\pm 7 \%$ ) containing both outer root sheath (AE13 ${ }^{+}$hair cortex, $\mathrm{AE}^{+} 5^{+}$medulla $)^{36,37}$, and inner root sheath $\left(\mathrm{AE} 15^{+}\right)^{37}$ struc-

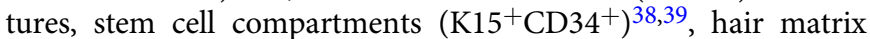
cells $\left(\mathrm{Shh}^{+}\right.$) (Fig. 3g, n and Supplementary Fig. 5). Neogenic hair follicles also maintained an adjoining dermal sheath $\left(\mathrm{SMA}^{+}\right.$, SM22 $\left.\alpha^{+}\right)^{40}$, biochemically distinct from DP $\left(\operatorname{Noggin}^{+}\right)^{25}$ (Supplementary Fig. 5). We also frequently observed $\mathrm{AP}^{+} \mathrm{DP}$ structures without accompanying hair germs despite their close proximity to overlying epidermis (Fig. 3j). Indeed, there were almost twice as many $\mathrm{AP}^{+} \mathrm{DP}$ as $\mathrm{K}^{+} 7^{+}$hair germs, suggesting that many DP $(42 \pm 15 \%)$ formed without establishing epithelial-mesenchymal interactions that could promote HF morphogenesis (Fig. 3f). DP formation without hair germ formation is not observed in normal endogenous hair follicle development in embryo or in adult large wounds ${ }^{11}$. These regenerative events were observed following wound closure. Intriguingly, even in the experiments where we induced $\mathrm{Hh}$ activation early during wound healing, the time of wound closure, proliferation, epidermal differentiation, AP distribution, angiogenesis and infiltration of immune cells including macrophages into the wound site were not altered prior to wound closure (Supplementary Fig. 6b-i).

Hh activation shifts dermal fibroblast fate toward DP. To understand whether SM22 ${ }^{+}$dermal cells universally induce the DP fate upon $\mathrm{Hh}$ activation, we isolated Tomato $^{+}$cells from wound dermis of SM22-rtTA; tetO-Cre; R26-SmoM2/Tomato (SM22-SmoM2) and SM22-rtTA; tetO-Cre; R26-Tomato (control) mice 3 days after complete re-epithelialization (dox treatment from PW1d to PW12d) and compared their molecular signatures by single-cell RNA sequencing (scRNA-seq) (Fig. 4). We performed unsupervised clustering with $\mathrm{K}$ means based on differentially expressed genes (DEGs), using tSNE ( $t$-distributed stochastic neighbor embedding). Based on expression of lineage markers for different cell types, we identified cellular clusters of fibroblasts, muscle cells, schwann cells, endothelial cells and immune cells (Fig. 4a-f $)^{41-44}$. Examination of Hh pathway mediators Gli1, Gli2, Ptch1, and Ptch2 showed that Hh pathway components were mainly expressed in the fibroblast cluster (Fig. 4g) and were largely restricted to the SM22-SmoM2 group (Fig. 4h, i). This is consistent with the lack of Gli1-lacZ expression in the wild-type dermis of small wounds (Fig. 1a and Supplementary Fig. 1h). The Hh-active fibroblast clusters showed upregulation of DP signature genes including Heyl, Sema6a, Wif1, Cxcr4, Ggta1, Hck, Snrpn and Rasd1 as previously defined by several groups (Fig. 4j) ${ }^{26,45-48}$, suggesting that $\mathrm{Hh}$ activation in $\mathrm{SM} 22^{+}$myofibroblasts are sufficient to globally induce upregulation of DP signature genes. Nonetheless, within this Hh-activated fibroblast population, we identified a divergence in the number and level of DP signature genes (Hh-active I and II), suggesting that $\mathrm{Hh}$-independent mechanisms are also involved in the regulation of DP signature genes. For example, while the upregulation of $B m p 3$ and $P l k 2$, known DP signature genes, was widely observed among $\mathrm{Hh}$-active fibroblasts (Hhactive I and II), expression of $A l p l(A P)$ and Lefl, vital markers for DP identification in histochemical analyses, was limited to a small subpopulation of $\mathrm{Hh}$-active fibroblasts (Hh-active II) (Fig. 4j).

Hh activation in wound epidermis forms BCC-like structure. In contrast to dermis-specific activation of the $\mathrm{Hh}$ pathway, forced Hh activation solely in epithelial cells (K14-CreER; R26SmoM2, TAM administration from PW1d to PW30d) did not promote DP formation in the wound area (Supplementary Fig. 7). 
a

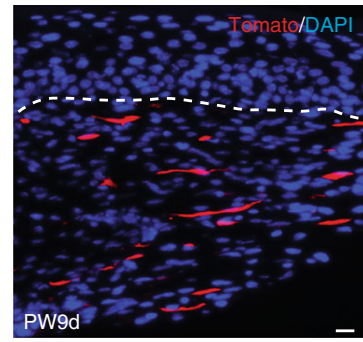

b

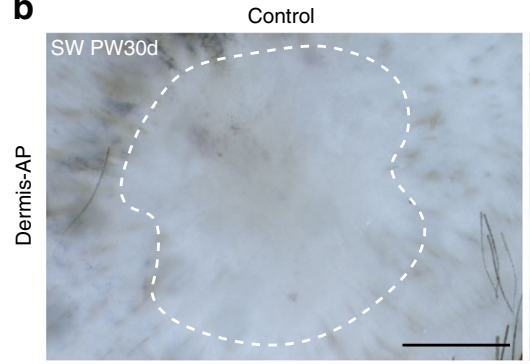

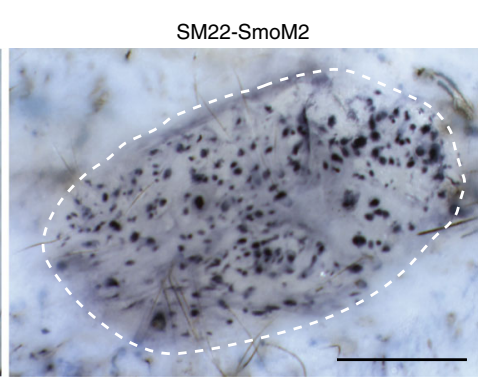

e

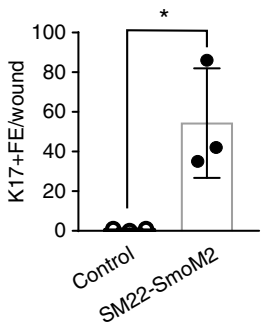

c

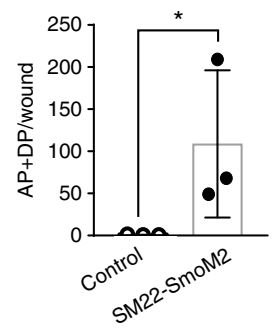

g
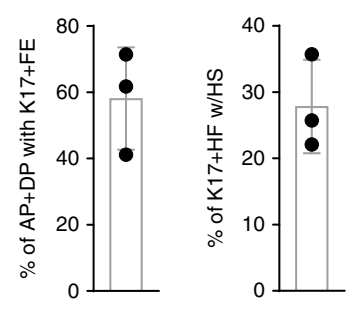

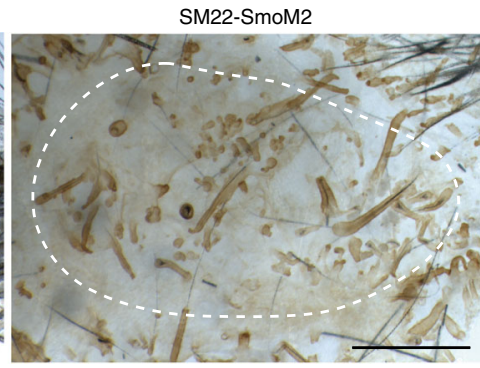

h

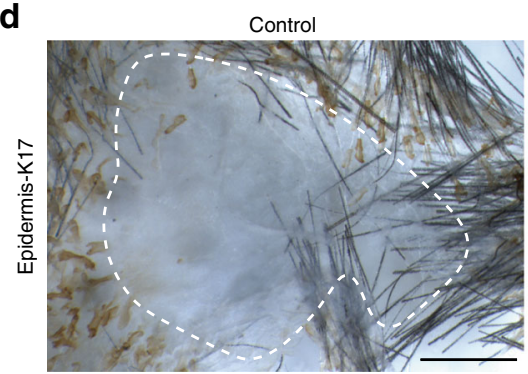

SM22-SmoM2

Control

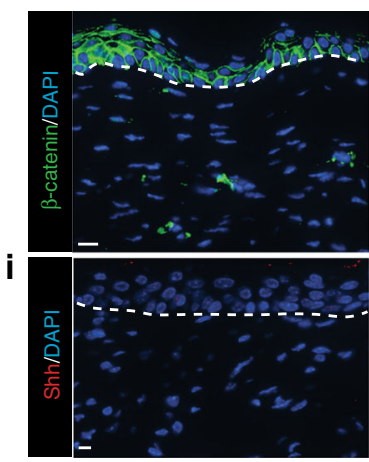

SM22-SmoM2

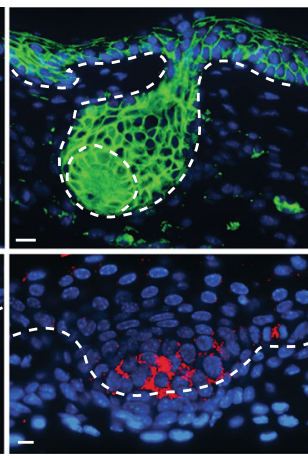

SM22-SmoM2

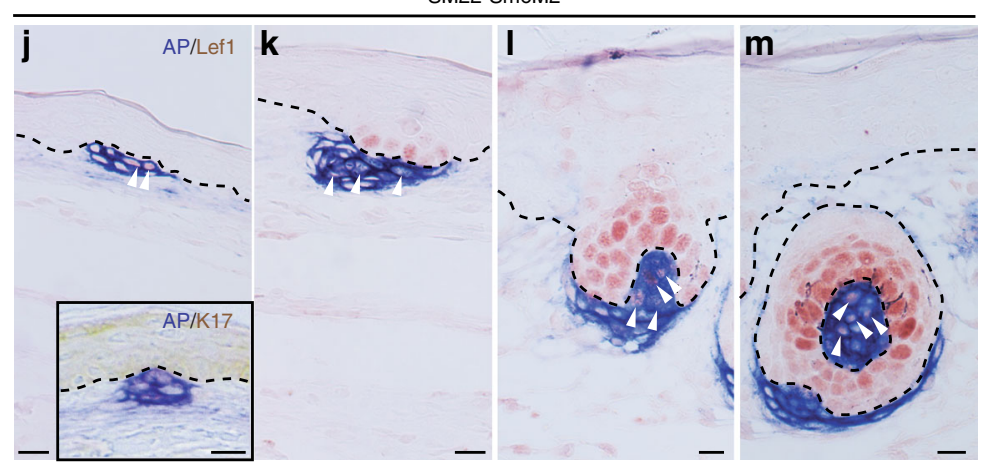

SM22-SmoM2
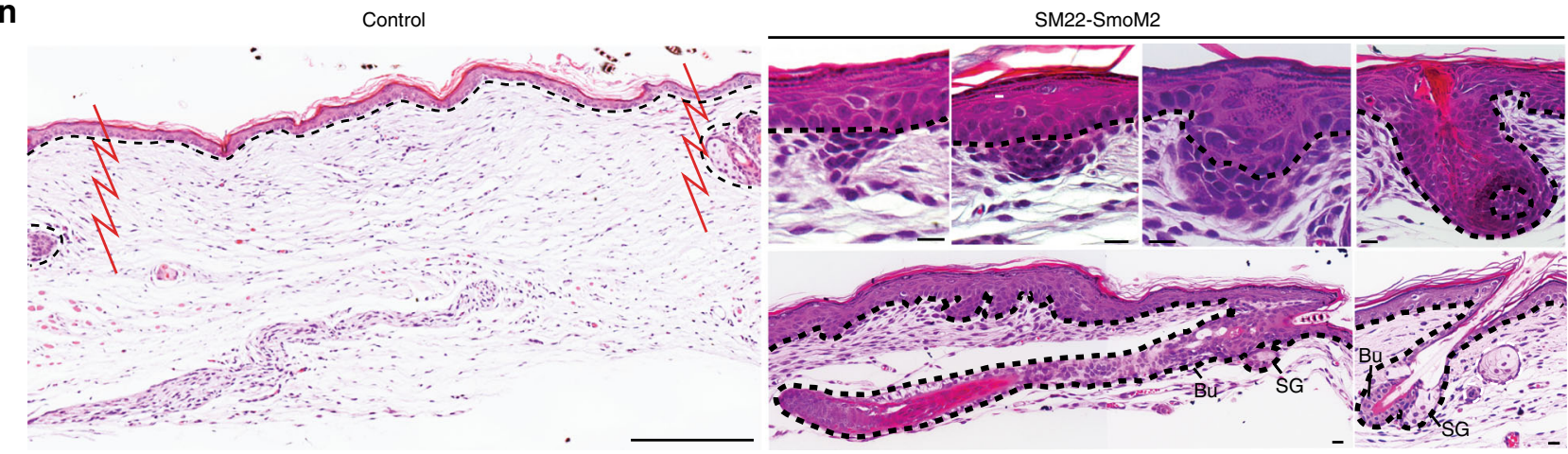

Fig. 3 Dermal Hh activation is sufficient to promote HFN in non-regenerative wounds. a Detection of tomato reporter on SW of SM22-rtTA; tetO-Cre; R26Tomato mice at indicated time. b-n SM22-rtTA; tetO-Cre: R26-SmoM2 (SM22-SmoM2) and littermate controls were subjected to SW and treated with doxycycline from PW1d until tissue harvest at PW30d ( $n=18 \mathrm{~W}(4-5 \mathrm{M})$ per condition). Whole-mount HFN assay (b, d) and quantifications (c, e). Percentage of $\mathrm{AP}^{+} \mathrm{DP}$ with $\mathrm{K} 17^{+} \mathrm{FE}(\mathbf{f})$. Percentage of $\mathrm{K} 17^{+} \mathrm{HF}$ with hair shaft $(\mathrm{HS})(\mathbf{g})$. Immunohistochemistry with indicated markers $(\mathbf{h}-\mathbf{m})$ and $\mathrm{H} \& \mathrm{E}$ (n). $n$ : number of wounds (W) or mice (M), Data are represented as mean \pm s.d., ${ }^{*} p<0.05$; Student's $t$-test, Dashed white circle: wound boundary, Dashed line: epidermis-dermis border, SW small wound, PW post-wound, DP dermal papilla, AP alkaline phosphatase, FE follicular epithelium, HF hair follicle, HS hair shaft, Bu bulge stem cell area, SG sebaceous gland. Scale bars represent $500 \mu \mathrm{m}$ (b, d), $100 \mu \mathrm{m}$ (n (Control)), $10 \mu \mathrm{m}(\mathbf{a}, \mathbf{h}-\mathbf{m}, \mathbf{n}(\mathrm{SM} 22-\mathrm{SmoM} 2)$ )

As previously observed, the epidermis of these wounds maintained numerous $\mathrm{K}^{+} 7^{+}$epithelial invaginations resembling BCCs without signs of hair follicle differentiation ${ }^{49-51}$. Also, the Hhdriven, BCC-like epithelial growths were not accompanied by underlying $\mathrm{DP}^{52}$.
Hh activation converts Wnt-active wound fibroblasts into DP. Next, given that Wnt signaling plays an essential role in hair follicle development and neogenesis ${ }^{11,53-56}$, we sought to examine the relationship between Wnt and Shh signaling. Our scRNA-seq analyses in myofibroblasts and examination of Axin2 expression 
in wounds from Axin2-LacZ mice both showed Wnt activity in scarring dermis of small wounds. scRNA-seq analyses demonstrated the expression of Axin2, Wls, and canonical Wnt ligands such as Wnt2 and Wnt10a (Fig. 5a, b). Our findings are consistent with previous reports that long-term Wnt signaling within dermal fibroblasts correlates with injury-induced fibrosis ${ }^{57,58}$. We hypothesized that dermal Wnt signaling is not sufficient to induce HFN without $\mathrm{Hh}$ activation. Indeed, constitutive activation of Wnt a

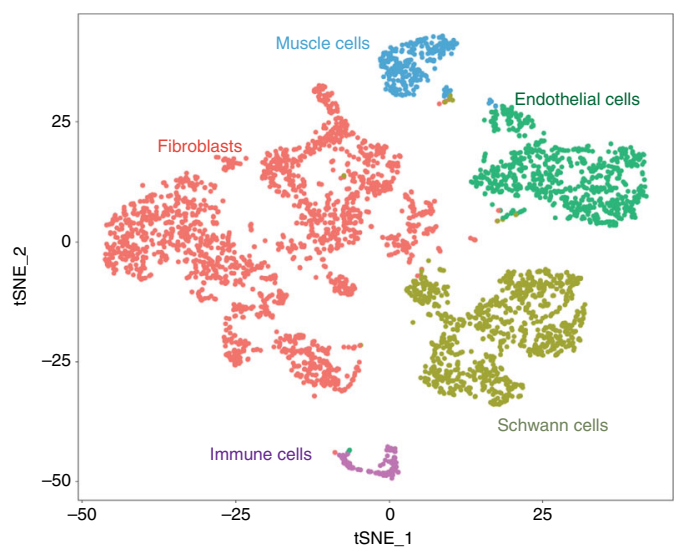

d Endothelial cells

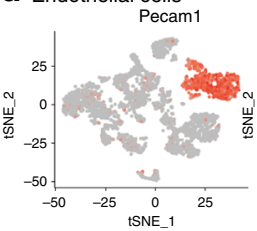

f Immune cells

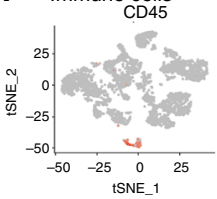

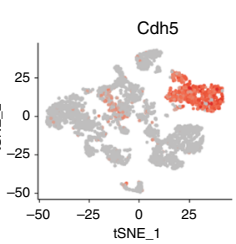

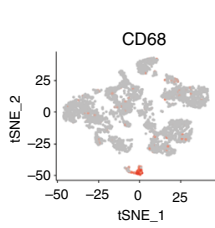

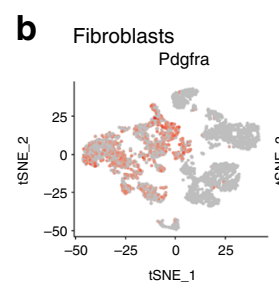
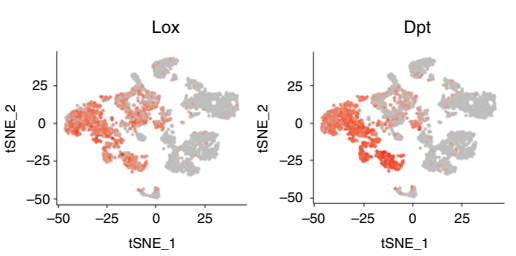

C Muscle cells
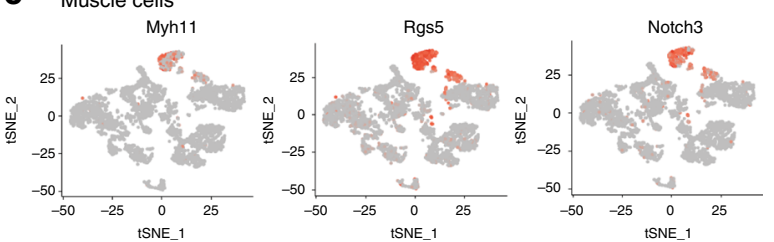

e Schwann cells
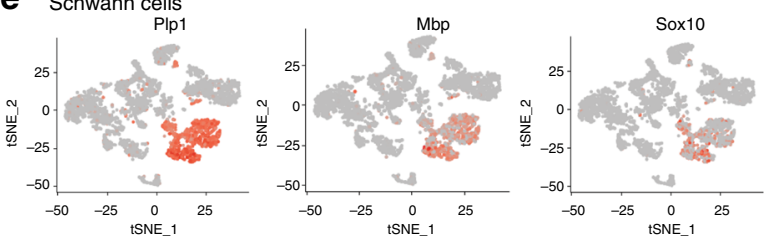

g

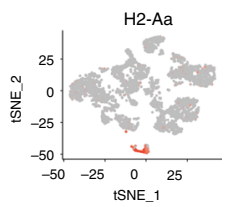

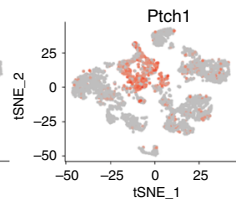

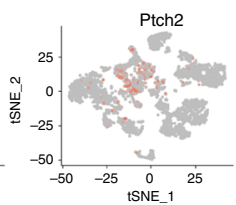

Fibroblasts
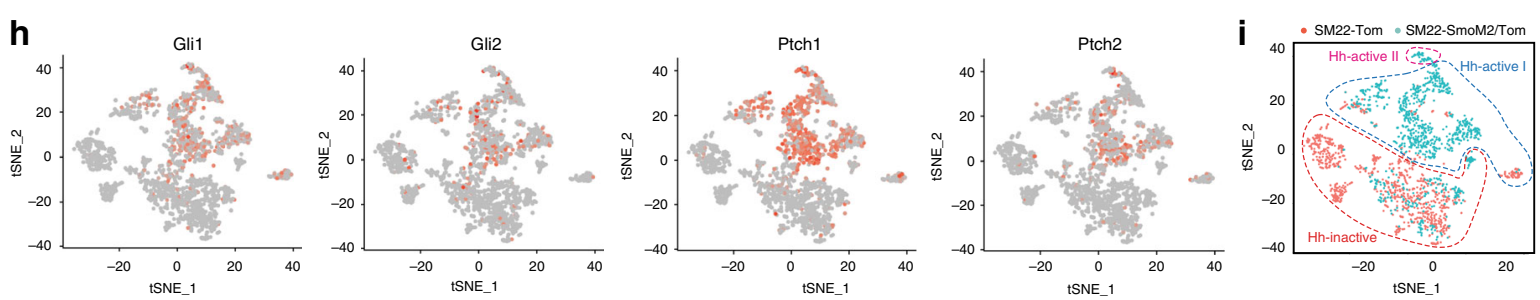

j

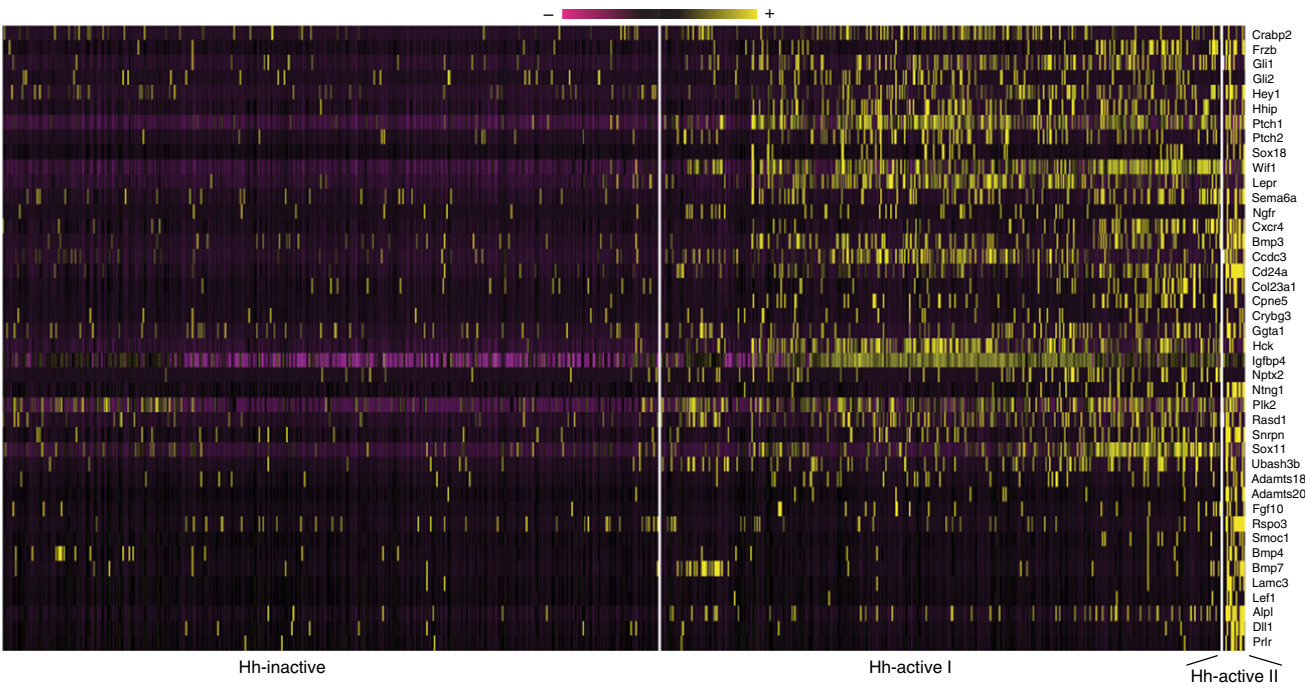


signaling (SM22-rtTA; tetO-Cre; $\beta$-catenin ${ }^{\text {ex } 3 / f l}$ ) in SM22+ dermal cells of small wounds did not promote new DP regeneration or HFN (Fig. 5c-h). However, dermal depletion of Wls, which is essential for Wnt ligand secretion, inhibited HFN in large wounds (SM22-rtTA; tetO-Cre; $W l_{s}{ }^{f l / f l}$ and dox treatment from PW3d to PW21d). These results suggest the requirement of dermal Wnt ligands for HFN (Fig. 5i-l).

We then sought to test whether Hh activation in dermal cells was capable of converting Wnt-active dermal fibroblasts to the DP fate. We induced forced Hh activation in Wnt responsive cells within small wounds, using the promoter of Axin2, a wellestablished target of canonical Wnt activity ${ }^{59,60}$ (Axin2-CreER; R26-SmoM2). TAM was administered into control and Axin2CreER; R26-SmoM2 from PW1d to PW30d. Smo overexpression in Wnt-active myofibroblasts resulted in extensive DP formation in small wounds (Fig. 6a-d). Genetic tracing of Axin2 ${ }^{+}$cells during HFN in reporter Axin2-CreER; R26-Tomato mice showed tomato expression in de novo DPs establishing that Axin2 $2^{+}$cells in wounds form DPs (Fig. 6e). In contrast, control wounds without $\mathrm{Hh}$ activation in Wnt-active dermal cells underwent scarring without HFN (Fig. 6a-d). These results show that Hh activation in Wnt-active dermal cells promotes their fate conversion into $\mathrm{DP}$, the regenerative dermal niche for $\mathrm{HF}$ formation.

\section{Discussion}

Our results demonstrate that wound repair can be redirected to promote regeneration following injury by modifying a key dermal signal (Fig. 6f). This study provides definitive evidence for a longheld concept that dermal cells are key components in determining wound healing outcome ${ }^{8}$. Our results suggest that the suppression of skin appendage regeneration in wound healing is due to the absence of dermal regeneration signals rather than intrinsic lack of regenerative competence in scarring cells. Installing developmental signals in the wound dermis may be a reasonable strategy to achieve regenerative healing in mammals. Epithelial activation of Wnt and Shh signaling was previously identified as critical for hair regeneration; however, these same pathways may also induce skin epithelial cancers when experimentally or pharmacologically augmented ${ }^{61,62}$. Our basic study with preclinical mouse models suggests that the capacity to create ectopic de novo DP in vivo by modulation of specific signals in the dermis may overcome this barrier and bring us closer to true skin renewal after injury.

Fibrotic scarring and epimorphic regeneration are frequently considered to be on opposite ends of the wound healing spectrum. This concept underpins attempts to promote regenerative healing by suppression of scarring mechanisms ${ }^{8,63}$. Our study suggests that scarring/fibrosis in skin wounds may not affect HF morphogenesis if the appropriate regenerative ques are applied. Although long-term activation of Wnt signaling, a hallmark of fibrotic repair, was observed in small wounds, the physiological level of Wnt signaling in scarring wounds did not negatively impact HFN in the presence of Shh activation. These studies provide evidence that fibrotic repair can be genetically subverted and offers possible tools to bypass extensive reprograming of adult skin cells into an "embryonic status" or for "stem cell transplantation" strategies to lead to regenerative healing in mammals.

HFN is largely a recapitulation of hair follicle development. However, the requirements for Shh regulation may be different in embryonic HF development and adult HFN. Shh-null mice can develop $\mathrm{DP}^{16}$ and Smo is dispensable for the initial establishment of DP in embryos ${ }^{17}$. Similarly, while epithelial Wnts are sufficient for embryonic HF development, both epithelial and dermal Wnts are needed for adult HFN ${ }^{54,64}$. The absolute requirement for Shh/Smo signaling in adult de novo DP formation may reflect a vulnerability in regeneration mechanisms compared to organogenesis in the embryo, whose development is often ensured by redundant compensatory mechanisms.

In conclusion, our study is the first to show that de novo DP can be created in adult skin by modulating a signaling pathway in the dermis. Future studies will be directed toward understanding the upstream mechanisms of Shh regulation, as well as downstream mechanisms leading to HF morphogenesis following de novo establishment of DP.

\section{Methods}

Mice. All animal protocols were approved by the Institutional Animal Care and Use Committee (IACUC) at NYU School of Medicine. All mice with proper genotype were used for the designed experiments regardless of sex. LSL-Shh mice in which mouse Shh is expressed under the control of $\beta$-actin promoter upon Cre medicated excision of stop sequence, were previously generated as described in Wang et $\mathrm{al}^{23}$. Axin2-CreER ${ }^{59}$ and $\beta$-catenin $f($ ex 3$) 65$ were obtained from indicated researchers. Gli1-LacZ (008211), R26-SmoM2 (005130), K14-CreER (005107), Shh flfl (004293) Pdgfra-CreER (018280), Smo fl/fl (004526), SM22-rtTA (006875), tetO-Cre (006224),

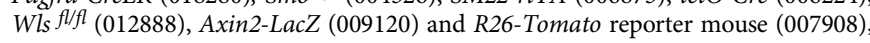
were purchased from the Jackson laboratory.

To induce CreER activity, tamoxifen (TAM) treatment was performed by intraperitoneal injection $(0.1 \mathrm{mg}$ per g body weight $)$ of a $20 \mathrm{mg}$ per $\mathrm{ml}$ solution in corn oil ${ }^{66}$. To induce $r t T A$ activity, mice were administered doxycycline-containing chow (20 g per kg, Bio-Serv).

Wound experiment. Wound experiments were carried out with 3-4-week-old or 7-8-week-old mice as described in Ito et al. ${ }^{11}$. All wounding experiments were performed after anesthetization of mice with isoflurane. Briefly, for fullthickness large wound, $1 \mathrm{~cm}^{2}(1 \times 1 \mathrm{~cm})$ or $2.25 \mathrm{~cm}^{2}(1.5 \times 1.5 \mathrm{~cm})$ of skin were excised for 3-4-week or 7-8-week-old mice, respectively. For small wound, skin was excised by $4 \mathrm{~mm}$ full-thickness biopsy punch (Acuderm Inc.) as published ${ }^{67}$. For loss of function study of Hh pathway, wounds were harvested at PW21d by which the number of neogenic HFs was saturated in control wounds. For gain of function study of Hh pathway, samples were harvested around 30 days after wounding (PW30d ) to allow continuous formation of neogenic HFs.

Whole-mount HFN assay. Whole-mount HFN assay to detect $\mathrm{K} 17^{+}$hair follicles and $\mathrm{AP}^{+} \mathrm{DP}$ was performed as previously described ${ }^{11}$. To analyze hair follicle (dermal papilla and follicular epithelium) regeneration after wounding, wounded skin was harvested from the mice and incubated in $5 \mathrm{mM}$ or $20 \mathrm{mM}$ EDTA in PBS at $37^{\circ} \mathrm{C}$ for $30 \mathrm{~min} \sim 2 \mathrm{~h}$ or overnight. The epidermis was gently separated from the dermis under a dissecting microscope (Axiovision Discovery V12, Zeiss, Germany). Both epidermis and dermis were fixed in $4 \%$ paraformaldehyde for $10 \mathrm{~min}$ at room temperature (RT) and rinsed with PBS. For the epidermis, standard DAB immunohistochemistry (see below) was performed with anti-K17 antibody (ab) (Abcam, 1:500). For the dermis, AP staining was performed. Dermis was incubated in NTMT solution $(100 \mathrm{mM} \mathrm{NaCl}, 100 \mathrm{mM}$ Tris-Cl ( $\mathrm{pH} 9.5$ ), $50 \mathrm{mM} \mathrm{MgCl}, 0.1 \%$ Tween-20) for $10 \mathrm{~min}$ and then incubated

Fig. 4 Dermal Hh activation induces DP fate in wound fibroblasts. scRNA-seq was performed with Tomato ${ }^{+}$cells isolated from wound dermis of both SM22-rtTA; tetO-Cre; R26-SmoM2/Tomato (SM22-SmoM2) and SM22-rtTA; tetO-Cre; R26-Tomato (control) mice 3 days after complete re-epithelialization. The mice were subject to SW and treated with doxycycline from PW1d until PW12d ( $n=12-25 \mathrm{~W}(3-5 \mathrm{M})$ per condition). a-i tSNE plots of 4680 SM22 + dermal cells split between control and Hh activated conditions. tSNE plot of SM22+ dermal cells colored by assigned lineages (a). tSNE plot of SM22+ dermal cells according to lineage-specific markers (b-f). tSNE plot of SM22+ dermal cells according to Hh pathway components (g). tSNE plot of SM22+ myofibroblasts according to Hh pathway components (h). tSNE plot of SM22+ myofibroblasts according to cell origin (i). $\mathbf{j}$ Heatmap showing the expression of DP signature genes. Yellow and black/purple correspond to high and low expression levels, respectively 
a
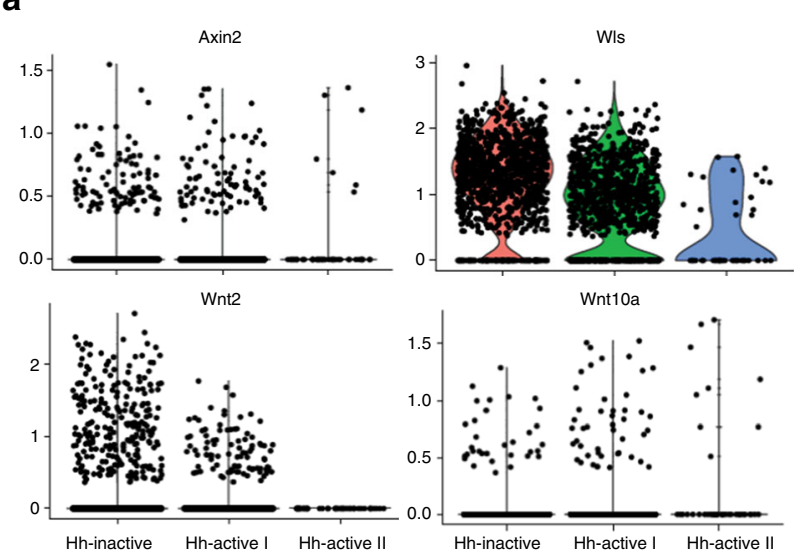

C
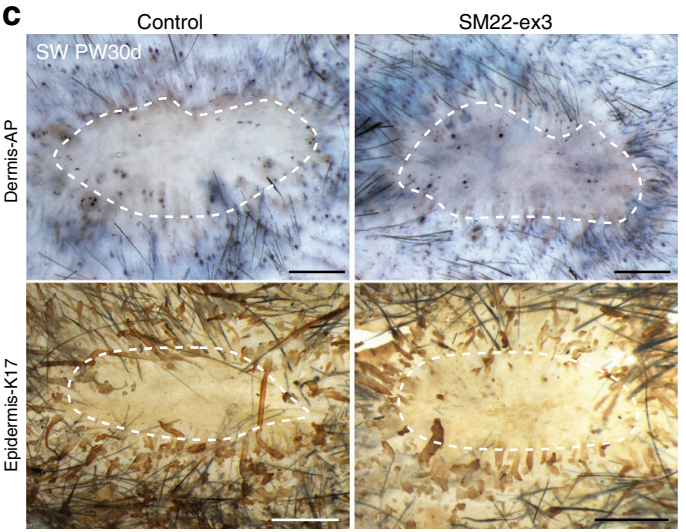

b
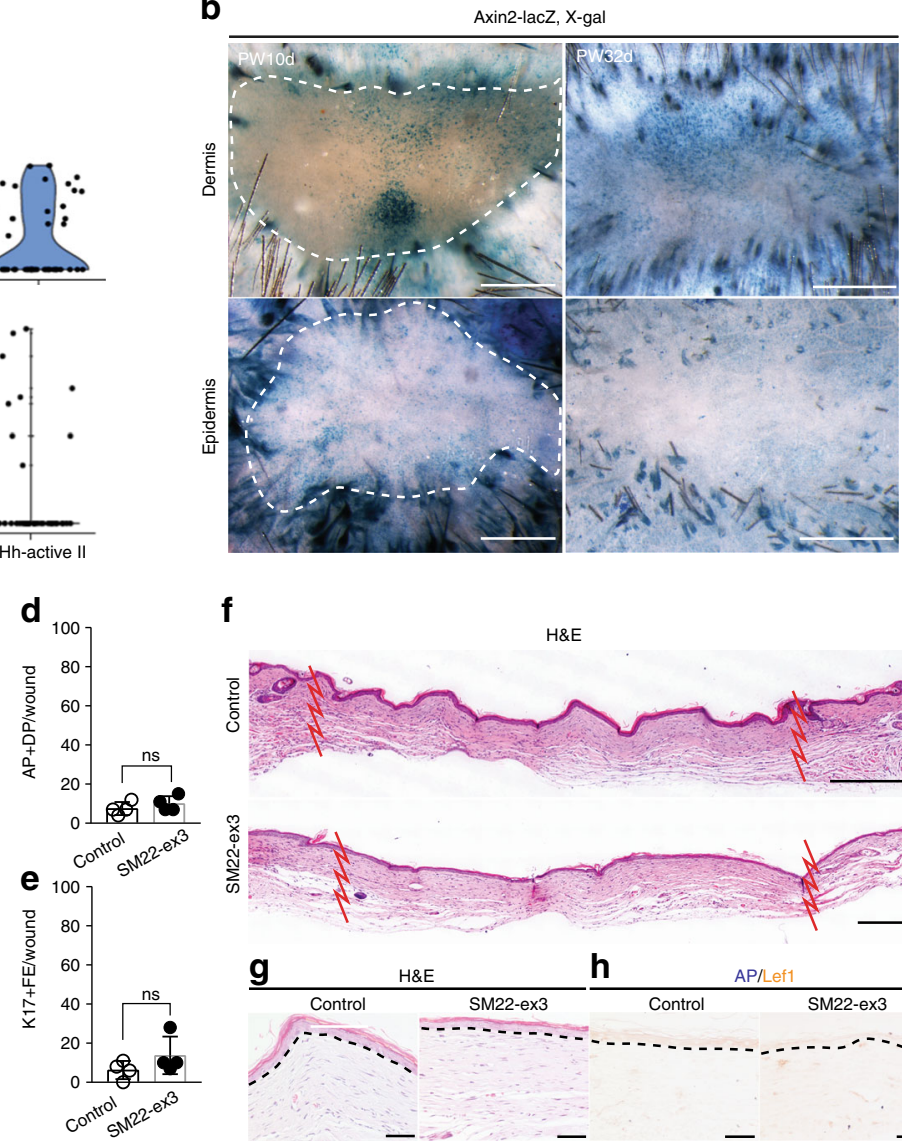

$\mathbf{f}$
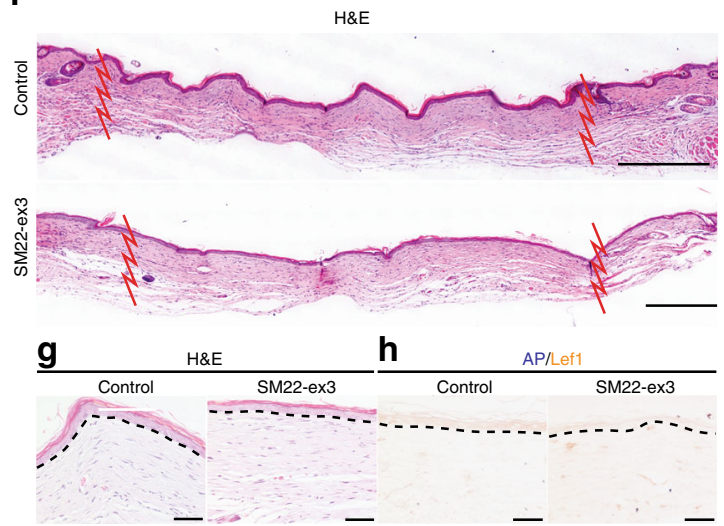
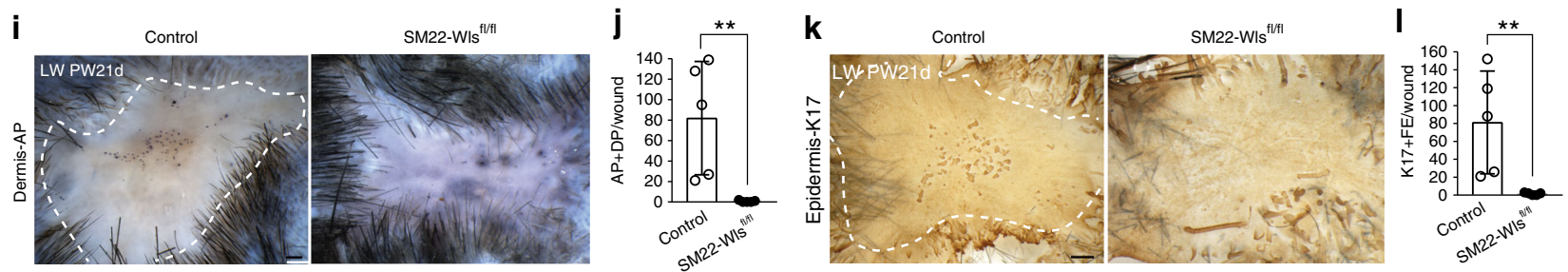

Fig. 5 Dermal Wnt activation alone is not sufficient for HFN. a Violin plots of Wnt pathway-related genes in SM22 ${ }^{+}$myofibroblasts. $\mathbf{b}$ X-gal staining in epidermis and dermis of SW of Axin2-LacZ mice at PW10d and PW32d. c-h SM22-rtTA; tetO-Cre; $\beta$-catenin fl(ex3)/+ (SM22-ex3) and littermate controls were subjected to SW and treated with doxycycline from PW1d until tissue harvest at PW30d ( $n=3-4 \mathrm{~W}$ (3-4 M) per condition). Whole-mount HFN assay (c) and quantifications (d, e). H\&E (f, $\mathbf{g})$ and AP/Lef1 staining (h) show lack of hair germ (HG) formation by $\beta$-catenin activation in dermis. $\mathbf{i}-\mathbf{I}$ SM22rtTA; tetO-Cre; W/s $\mathrm{fl} / \mathrm{fl}(\mathrm{SM} 22-\mathrm{W} / \mathrm{s} / \mathrm{fl} / \mathrm{fl})$ and littermate controls were subjected to $\mathrm{LW}$ and treated with doxycycline from PW3d until tissue harvest at PW21d ( $n=4-5$ W (4-5 M) per condition). Whole-mount HFN assay $(\mathbf{i}, \mathbf{k})$ and quantifications $(\mathbf{j}, \mathbf{l})$. $n$ : number of wounds (W) or mice (M), Dashed white circle and zigzag line: wound boundary, Dashed line: epidermis-dermis border, SW small wound, LW large wound, AP alkaline phosphatase, PW postwound, Scale bars represent $500 \mu \mathrm{m}(\mathbf{b}, \mathbf{c}, \mathbf{i}, \mathbf{k}), 100 \mu \mathrm{m}(\mathbf{f}), 50 \mu \mathrm{m}(\mathbf{g}, \mathbf{h})$

in NTMT containing in NBT/BCIP (Roche, 1:50) solution at RT until color was visualized.

Histochemistry. Immunohistochemistry was performed as published ${ }^{66}$. For paraffin sections, tissues were fixed in $4 \%$ PFA at $4{ }^{\circ} \mathrm{C}$ overnight and rinsed with PBS. Following sequential dehydration in ethanol and xylene, tissues were infiltrated by paraffin and embedded in fresh paraffin. The paraffin-embedded tissue blocks were chilled on ice for $10-15 \mathrm{~min}$ and cut at $6 \mu \mathrm{m}$ thickness. The $6 \mu \mathrm{m}$ tissue slices were flattened out on warm water and placed on microscope slides and dried out at $37^{\circ} \mathrm{C}$ overnight. The tissue sections were rehydrated through xylene and graded series of ethanol $(2 \times 100 \%, 90 \%, 80 \%, 70 \%$, and $50 \%)$ and rinsed with PBS. After antigen retrieval in Tris-EDTA (pH 8.0), the tissue sections were incubated with blocking solution (10\% BCS in PBS containing $0.1 \%$ Tween-20) for $1 \mathrm{~h}$ at RT then appropriate primary antibodies at RT for $2 \mathrm{~h}$ or $4^{\circ} \mathrm{C}$ overnight. After PBS washing, the tissue sections were incubated with secondary antibodies at RT for $1 \mathrm{~h}$. If necessary, the second and third primary antibodies were used on the same tissue sections with corresponding secondary antibodies. Following PBS rinsing, the stained slides were mounted with mounting medium and stored at $4{ }^{\circ} \mathrm{C}$ for analysis. For frozen section, tissues were fixed in $4 \%$ PFA on ice for $10 \mathrm{~min}$ and embedded in OCT compound on dry ice. The frozen blocks were cut at $10 \mu \mathrm{m}$ thickness, placed on microscope slides, and dried out at RT. After PBS washing, the tissue sections were incubated in blocking, primary, and secondary ab solutions as described above. All immunohistochemical analysis was observed and photographed on an upright Nikon Eclipse Ti or Zeiss Axiophot microscopes. Following antibodies were used: rabbit anti-K17 (1:500, Abcam), rabbit anti-Lef1 (1:100, Cell signaling), rabbit anti-Noggin (1:100, Abcam), rabbit anti-RFP (1:500, Rockland), rabbit anti-Shh (1:50, Santa Cruz), rabbit anti-SMA (1:100, Thermo Scientific), rabbit anti-F4/80 (1:100, Cell signaling), chicken anti-K14 (1:500, BioLegend), mouse anti- $\beta$-catenin (1:500, Sigma), mouse anti-SM22a (1:100, Abcam), mouse anti-AE13 (1:20, a gift of T.T. Sun), mouse anti-AE15 (1:20, a gift of T.T. Sun), mouse anti-K15 (1:100, NeoMarkers), and rat anti-CD34 (1:50, BD Bioscience). For histology, paraffin sections were stained with hematoxylin and eosin in accordance with a general method. To detect collagen protein, trichrome staining and picrosirius red staining were carried out using Masson's Trichrome Stain Kit and Picrosirius Red Stain Kit, respectively (Polysciences). Trichrome staining was performed at NYUMC experimental pathology core. 
a
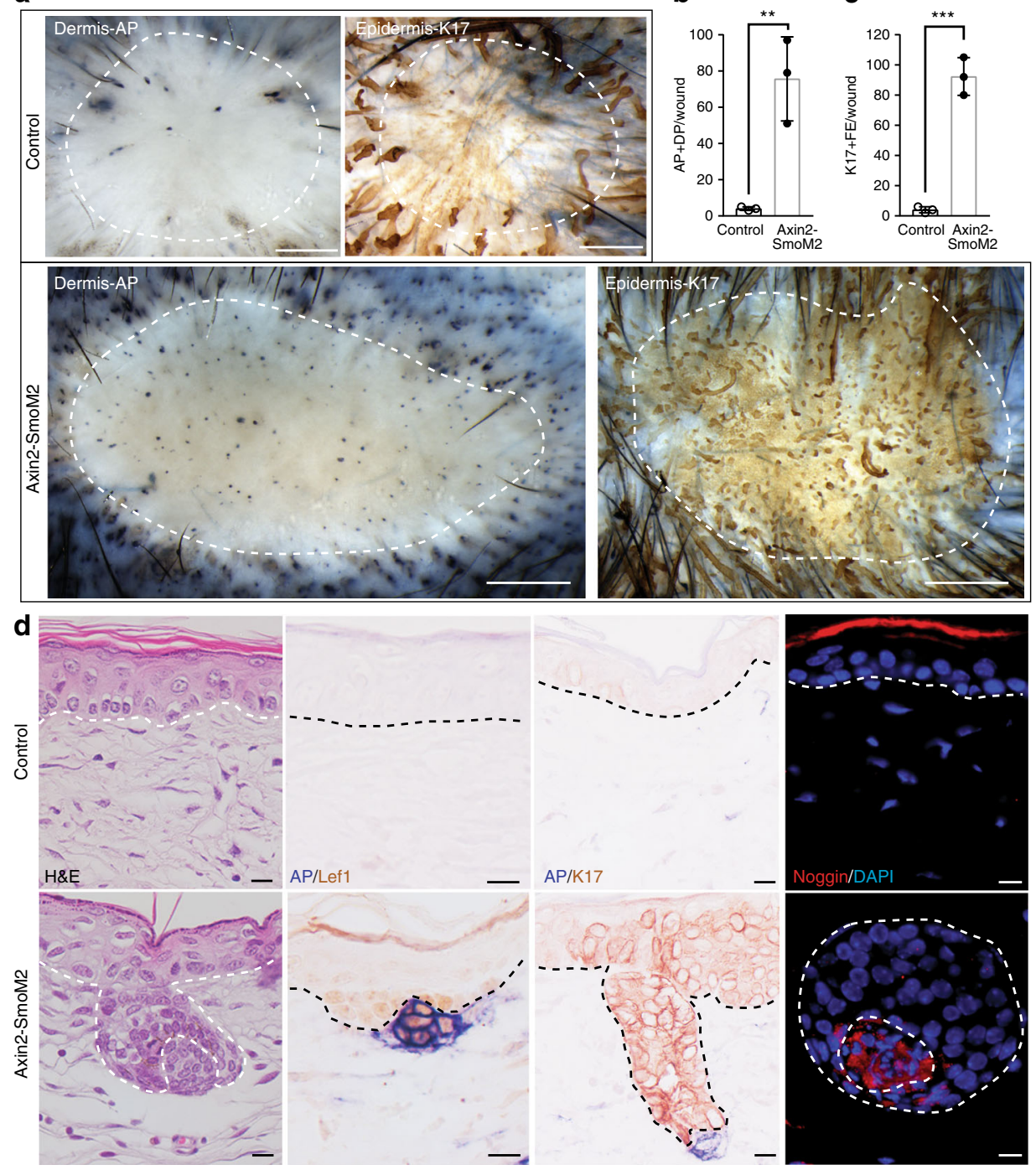

e
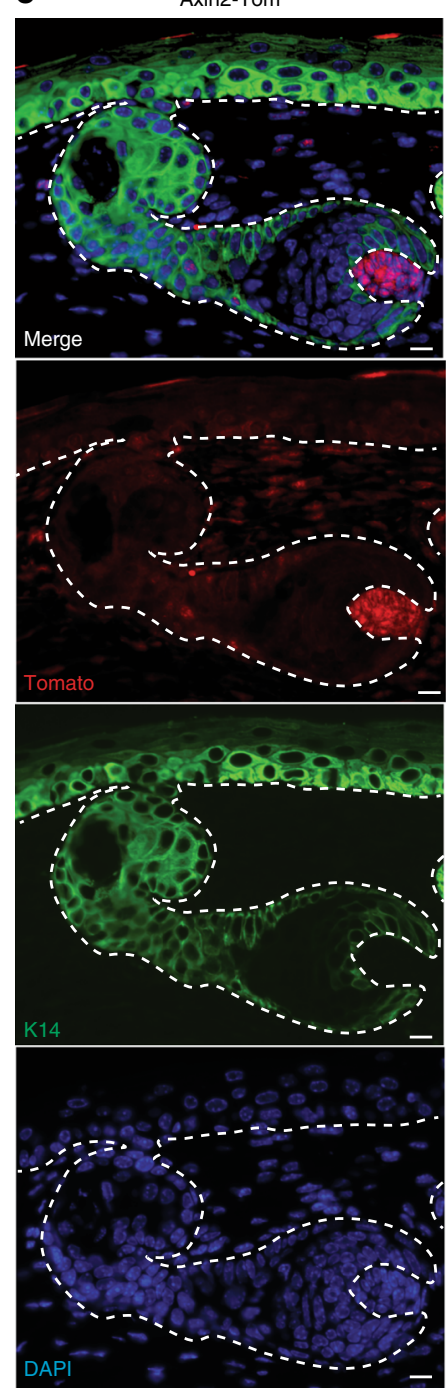

$\mathbf{f}$

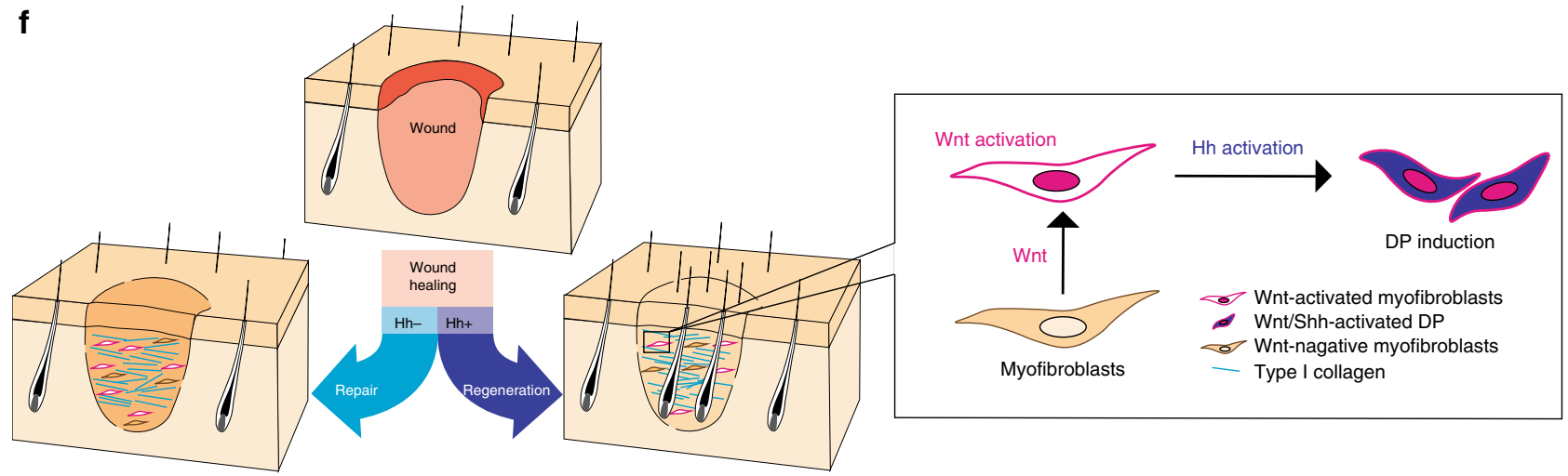

Fig. $6 \mathrm{Hh}$ activation can convert fibrotic Wnt-active dermal cells into DP in wounds. a-d Axin2-CreER; R26-SmoM2 (Axin2-SmoM2) and littermate controls were subjected to SW and treated with TAM from PW1d until tissue harvest at PW30d ( $n=9-15 \mathrm{~W}$ (3-5 M) per condition). Whole-mount HFN assay (a) and quantifications $(\mathbf{b}, \mathbf{c})$. H\&E and immunohistochemical analyses with indicated markers (d). e Tracing of tomato-labeled Axin2 ${ }^{+}$cells in Axin2-CreER; $R 26-$ Tomato (Axin2-Tom). Axin2-Tom mice were subjected to LW and treated with TAM from PW3d until PW12d (before complete re-epithelialization). Tissue was harvested at PW23d and stained with anti-RFP antibody. $\mathbf{f}$ Model: Conversion of wound repair to regeneration in adult skin. Wound healing in mammalian skin typically results in scarring and lack of appendage regeneration. Dermal Hh activation can install de novo dermal papilla into wounds, resulting in regenerative $\mathrm{HF}$ neogenesis, despite collagen deposition in adult wounds. $n$ : number of wounds $(W)$ or mice (M), Data are represented as mean \pm s.d., ${ }^{\star *} p<0.01,{ }^{\star \star \star} p<0.001$; Student's $t$-test, Dashed white circle: wound boundary, Dashed line: epidermis-dermis border, SW small wound, LW large wound, PW post-wound, DP dermal papilla, AP alkaline phosphatase, FE follicular epithelium, HF hair follicle, Scale bars represent $500 \mu \mathrm{m}$ (a), $10 \mu \mathrm{m}$ (d, e) 
X-gal staining. X-gal staining was performed as published ${ }^{68}$. Skin wound tissues were fixed in $4 \%$ PFA at $4{ }^{\circ} \mathrm{C}$ for $30 \mathrm{~min}$ and rinsed with PBS. The tissues were incubated in X-gal rinse buffer $(2 \mathrm{mM} \mathrm{MgCl} 2,0.01 \%$ Sodium deoxycholate, and $0.02 \% \mathrm{NP}-40$ in PBS) for $10 \mathrm{~min}$ at RT and then in X-gal (5-bromo-4-chloro-3indolyl- $\beta$-d-galactopyranoside) staining buffer $\left(0.7 \mathrm{mg} / \mathrm{ml} \mathrm{X}\right.$-gal, $0.5 \mathrm{M} \mathrm{K}_{4} \mathrm{Fe}(\mathrm{CN})_{6}$ and $0.5 \mathrm{M} \mathrm{K}_{3} \mathrm{Fe}(\mathrm{CN})_{6}$ in X-gal rinse buffer) at $\mathrm{RT}$ or $37^{\circ} \mathrm{C}$ until color was visualized. The tissues were photographed in whole mount using a dissection microscope (Zeiss, Discovery V12). The tissues were then dehydrated in standard graded series of ethanol, embedded in paraffin blocks and cut into 6 - $\mu$ m-thick sections. To visualize nucleus, the tissues were counterstained with nuclear fast red solution. Small wounds of Axin2-LacZ mice were incubated in $20 \mathrm{mM}$ EDTA in PBS at $37^{\circ} \mathrm{C}$ for $1 \mathrm{~h}$ to separate dermis from epidermis before X-gal staining.

RNA-seq analysis. Skin wound tissues (PW11d) of control and K14-CreER; LSLShh mice were incubated in $20 \mathrm{mM}$ EDTA solution at $37^{\circ} \mathrm{C}$ for $30 \mathrm{~min}$. After separation of epidermis from dermis, total RNA was isolated from epidermis and dermis using RNeasy Plus Micro-Kit (Qiagen) as described by the manufacturer. Total RNA was provided to Genome Technology Center at NYU Langone Medical Center for preparing RNA-seq libraries and sequencing. RNA-seq libraries were prepared using the Illumina TruSeq Stranded Total RNA library prep, after ribodepletion with Ribozero Gold kit (Illumina) starting from 200 ng of DNAse I treated total RNA, following the manufacturer's protocol (15 cycles of PCR amplification). The amplified libraries were purified using AMPure beads, quantified by Qubit and QPCR, and visualized in an Agilent Bioanalyzer. The libraries were pooled equimolarly, and sequenced on two lanes of an Illumina HiSeq 2500 flow cell, v4 chemistry as paired-end 50. The differentially expressed genes (DEG) were submitted to DAVID for GO term analysis ${ }^{69}$. Top related enriched terms were selected and shown in the figures.

Single-cell RNA-seq analysis. Skin wounds were collected from of both $S M 22-$ rtTA; tetO-Cre; R26-SmoM2/Tomato (SM22-SmoM2) and SM22-rtTA; tetO-Cre; R26-Tomato (control) mice 3 days after complete re-epithelialization (dox treatment from PW1d to PW12d) and incubated in $20 \mathrm{mM}$ EDTA solution at $37^{\circ} \mathrm{C}$ for $30 \mathrm{~min}$ to separate dermis from epidermis. The separated dermis was incubated in Dulbecco's Modified Eagle Medium (DMEM, Corning) containing 10\% FBS (Corning) and $0.35 \%$ type I collagenase (Worthington) at $37^{\circ} \mathrm{C}$ for $1 \mathrm{~h}$. After rinsing with PBS, Tomato $^{+}$cells from isolated dermal cells were sorted with a FACSAria II cell sorter (BD biosciences). scRNA-seq libraries were prepared using the following: Single-Cell 3' Reagent Kits v2: Chromium ${ }^{\text {tw }}$ Single-Cell 3' Library \& Gel Bead Kit v2 PN-120237, Single-Cell 3' Chip Kit v2 PN-120236, i7 Multiplex Kit PN-120262" (10x Genomics) 46 and the Single-Cell 3' Reagent Kits v2 User Guide (Manual Part \# CG00052 Rev A). Libraries were run on an Illumina HiSeq 4000 as $2 \times 150$ paired-end reads, one full lane per sample, for approximately $>90 \%$ sequencing saturation. Sequencing results were demultiplexed and converted to FASTQ format using Illumina bcl2fastq software. The Cell Ranger Single-Cell Software Suite (https://support.10xgenomics.com/single-cell-gene- expression/ software/pipelines/latest/what-is-cell-ranger) was used to perform sample demultiplexing, barcode processing, and single-cell $3^{\prime}$ gene counting. The cDNA insert was aligned to the mm10/GRCm 38 reference genome. Only confidently mapped, non-PCR duplicates with valid barcodes and UMIs were used to generate the genebarcode matrix. Further analysis and visualization was performed using Seurat, an $\mathrm{R}$ package containing implementations of commonly used single-cell analytical techniques, including the identification of highly variable genes, dimensionality reduction, standard unsupervised clustering algorithms, and the discovery of differentially expressed genes and markers (http://www.ncbi.nlm.nih.gov/pubmed/ 26000488).

Quantitative reverse transcription PCR (qRT-PCR). Total RNA was isolated using RNeasy Micro-Kit (Qiagen) as described by manufacturer and reversetranscribed with Superscript III First Strand Synthesis System (Invitrogen) for cDNA synthesis. cDNA was amplified using taqman probes and the ABI 7900HT SDS system. Transcripts were quantified relative to the housekeeping gene, GAPDH. The probes used for this study were listed in Supplementary Table 1.

Hydroxyproline assay. To measure collagen content, hydroxyproline assay kit (Sigma) was used according to manufacturer's protocol. Whole wound tissues were collected and homogenized in $100 \mu \mathrm{l}$ of water per $10 \mathrm{mg}$ tissue. After adding $100 \mu \mathrm{l}$ of $\mathrm{HCl}(\sim 12 \mathrm{M})$ per $10 \mathrm{mg}$ tissue into the homogenized tissue, the mixture was hydrolyzed at $120^{\circ} \mathrm{C}$ for $3 \mathrm{~h}$. A total of $1-2 \mu \mathrm{l}$ of supernatant was incubated in 100 $\mu \mathrm{l}$ of Chloramine T/Oxidation buffer mixture for $5 \mathrm{~min}$ and then, in $100 \mu \mathrm{l}$ of diluted DMAB reagent for $90 \mathrm{~min}$, sequentially. The absorbance was measured at $560 \mathrm{~nm}$ using SpectraMax M3 (Molecular Devices).

Transmission electron microscopy. Transmission electron microscopy (TEM) was carried out in Microscopy Laboratory at NYU Langone Medical Center ${ }^{70}$. The harvested wounds were dissected $(0.5 \mathrm{Xl} \mathrm{cm})$ and put the wounds on top of paper tower. The skin wounds were fixed in the fixative containing $2.5 \%$ glutaraldehyde, and $2 \%$ paraformaldehyde in $0.1 \mathrm{M}$ sodium cacodylate buffer $(\mathrm{pH} 7.2)$ with $1 \%$ tannic acid for $30 \mathrm{~min}$ and further dissected to $1 \times 3 \mathrm{~mm}$ smaller pieces. Fixation process was continued in the same fixative at RT for $2 \mathrm{~h}$, then $4{ }^{\circ} \mathrm{C}$ overnight. The skin then post-fixed with $1 \%$ osmium tetroxide for $2 \mathrm{~h}$ at RT, block staining in $1 \%$ uranyl acetate overnight at $4{ }^{\circ} \mathrm{C}$, then dehydration in a standard manner and embedded in EMbed 812 (Electron Microscopy Sciences, Hatfield, PA) for transmission electron microscopy. Semi-thin sections were cut at $1 \mathrm{~mm}$ and stained with $1 \%$ Toluidine Blue to evaluate the orientation of the sample. Ultrathin sections ( 60 $\mathrm{nm}$ ) were cut, mounted on copper grids and stained with uranyl acetate and lead citrate. Stained grids were examined under Philips $\mathrm{cm}$-12 electron microscope (FEI; Eindhoven, The Netherlands) and photographed with a Gatan (4k X2.7 k) digital camera (Gatan, Inc., Pleasanton, CA).

Statistical analysis and image processing. The whole-mount HFN assay was performed with at least three wound samples per genotype, and the data were representative of over three independent experiments. Data were represented as mean \pm s.d. To calculate $p$-values, Student's $t$-test was used on Microsoft Excel, with two-tailed tests and unequal variance. All graphs were generated by Microsoft Excel and GraphPad Prism. Images were processed using Image J and Adobe Photoshop. For transforming AP signals into colored dots using Photoshop, images of AP staining of wound were first turned into black and white, reduced background, and changed into red, green or cyan colors. Three images were then merged by overlapping the center of each wound.

\section{Data availability}

RNA-seq data and scRNA-seq have been deposited in the Gene Expression Omnibus (GEO) database under accession codes GSE94893 and GSE112671, respectively. All other data of this study are available from the corresponding author upon reasonable request.

Received: 27 June 2018 Accepted: 15 October 2018 Published online: 21 November 2018

\section{References}

1. Sennett, R. \& Rendl, M. Mesenchymal-epithelial interactions during hair follicle morphogenesis and cycling. Semin. Cell Dev. Biol. 23, 917-927 (2012).

2. Hardy, M. H. The secret life of the hair follicle. Trends Genet. 8, 55-61 (1992).

3. Morgan, B. A. The dermal papilla: An instructive niche for epithelial stem and progenitor cells in development and regeneration of the hair follicle. Cold Spring Harb. Perspect. Med. 4, a01518 (2014).

4. Rompolas, P. \& Greco, V. Stem cell dynamics in the hair follicle niche. Semin Cell Dev. Biol. 25-26, 34-42 (2014).

5. Cotsarelis, G., Sun, T. T. \& Lavker, R. M. Label-retaining cells reside in the bulge area of pilosebaceous unit: implications for follicular stem cells, hair cycle, and skin carcinogenesis. Cell 61, 1329-1337 (1990).

6. Blanpain, C. \& Fuchs, E. Epidermal homeostasis: a balancing act of stem cells in the skin. Nat. Rev. Mol. Cell Biol. 10, 207-217 (2009).

7. Schneider, M. R., Schmidt-Ullrich, R. \& Paus, R. The hair follicle as a dynamic miniorgan. Curr. Biol. 19, R132-R142 (2009).

8. Gurtner, G. C., Werner, S., Barrandon, Y. \& Longaker, M. T. Wound repair and regeneration. Nature 453, 314-321 (2008).

9. Seifert, A. W. \& Maden, M. New insights into vertebrate skin regeneration. Int. Rev. Cell Mol. Biol. 310, 129-169 (2014).

10. Leavitt, T. et al. Scarless wound healing: finding the right cells and signals. Cell Tissue Res. 365, 483-493 (2016).

11. Ito, M. et al. Wnt-dependent de novo hair follicle regeneration in adult mouse skin after wounding. Nature 447, 316-320 (2007).

12. Chuong, C. M. Regenerative biology: new hair from healing wounds. Nature 447, 265-266 (2007).

13. Straile, W. E. in Advances in Biology of Skin, Vol. 9 (eds Montagna, W. \& Dobson, R.) 369-391 (Pergamon Press, New York, 1967).

14. Chiang, C. et al. Essential role for Sonic hedgehog during hair follicle morphogenesis. Dev. Biol. 205, 1-9 (1999).

15. Ghali, L., Wong, S. T., Green, J., Tidman, N. \& Quinn, A. G. Glil protein is expressed in basal cell carcinomas, outer root sheath keratinocytes and a subpopulation of mesenchymal cells in normal human skin. J. Invest. Dermatol. 113, 595-599 (1999).

16. St-Jacques, B. et al. Sonic hedgehog signaling is essential for hair development Curr. Biol. 8, 1058-1068 (1998).

17. Woo, W. M., Zhen, H. H. \& Oro, A. E. Shh maintains dermal papilla identity and hair morphogenesis via a Noggin-Shh regulatory loop. Genes Dev. 26, 1235-1246 (2012)

18. Ouspenskaia, T., Matos, I., Mertz, A. F., Fiore, V. F. \& Fuchs, E. WNT-SHH Antagonism Specifies and Expands Stem Cells prior to Niche Formation. Cell 164, 156-169 (2016). 
19. Gao, J. et al. Laminin-511 is an epithelial message promoting dermal papilla development and function during early hair morphogenesis. Genes Dev. 22 2111-2124 (2008).

20. Sato, N., Leopold, P. L. \& Crystal, R. G. Induction of the hair growth phase in postnatal mice by localized transient expression of Sonic hedgehog. J. Clin. Invest. 104, 855-864 (1999).

21. Sato, N., Leopold, P. L. \& Crystal, R. G. Effect of adenovirus-mediated expression of Sonic hedgehog gene on hair regrowth in mice with chemotherapy-induced alopecia. J. Natl. Cancer Inst. 93, 1858-1864 (2001).

22. Morgan, B. A., Orkin, R. W., Noramly, S. \& Perez, A. Stage-specific effects of sonic hedgehog expression in the epidermis. Dev. Biol. 201, 1-12 (1998).

23. Wang, D. H. et al. Aberrant epithelial-mesenchymal Hedgehog signaling characterizes Barrett's metaplasia. Gastroenterology 138, 1810-1822 (2010).

24. Zhou, P., Byrne, C., Jacobs, J. \& Fuchs, E. Lymphoid enhancer factor 1 directs hair follicle patterning and epithelial cell fate. Genes Dev. 9, 700-713 (1995).

25. Botchkarev, V. A. et al. Noggin is a mesenchymally derived stimulator of hairfollicle induction. Nat. Cell Biol. 1, 158-164 (1999).

26. Sennett, R. et al. An Integrated Transcriptome Atlas of Embryonic Hair Follicle Progenitors, Their Niche, and the Developing Skin. Dev. Cell. 34, 577-591 (2015).

27. Merkel, J. R., DiPaolo, B. R., Hallock, G. G. \& Rice, D. C. Type I and type III collagen content of healing wounds in fetal and adult rats. Proc. Soc. Exp. Biol. Med. 187, 493-497 (1988)

28. Whitby, D. J. \& Ferguson, M. W. The extracellular matrix of lip wounds in fetal, neonatal and adult mice. Development 112, 651-668 (1991).

29. Collins, C. A., Kretzschmar, K. \& Watt, F. M. Reprogramming adult dermis to a neonatal state through epidermal activation of beta-catenin. Development 138, 5189-5199 (2011).

30. Seifert, A. W. et al. Skin shedding and tissue regeneration in African spiny mice (Acomys). Nature 489, 561-565 (2012).

31. Doillon, C. J., Dunn, M. G. \& Silver, F. H. Relationship between mechanical properties and collagen structure of closed and open wounds. J. Biomech. Eng. 110, 352-356 (1988).

32. West, J. et al. Pulmonary hypertension in transgenic mice expressing a dominant-negative BMPRII gene in smooth muscle. Circ. Res. 94, 1109-1114 (2004).

33. Wixler, V. et al. Deficiency in the LIM-only protein Fhl2 impairs skin wound healing. J. Cell Biol. 177, 163-172 (2007).

34. Plikus, M. V. et al. Regeneration of fat cells from myofibroblasts during wound healing. Science 355, 748-752 (2017).

35. Zhang, Y. et al. Activation of beta-catenin signaling programs embryonic epidermis to hair follicle fate. Development 135, 2161-2172 (2008).

36. Lynch, M. H., O'Guin, W. M., Hardy, C., Mak, L. \& Sun, T. T. Acidic and basic hair/nail ("hard") keratins: their colocalization in upper cortical and cuticle cells of the human hair follicle and their relationship to "soft" keratins. J. Cell Biol. 103, 2593-2606 (1986).

37. O'Guin, W. M., Sun, T. T. \& Manabe, M. Interaction of trichohyalin with intermediate filaments: three immunologically defined stages of trichohyalin maturation. J. Invest. Dermatol. 98, 24-32 (1992).

38. Liu, Y., Lyle, S., Yang, Z. \& Cotsarelis, G. Keratin 15 promoter targets putative epithelial stem cells in the hair follicle bulge. J. Invest. Dermatol. 121, 963-968 (2003).

39. Trempus, C. S. et al. Enrichment for living murine keratinocytes from the hair follicle bulge with the cell surface marker CD34. J. Invest. Dermatol. 120, 501-511 (2003).

40. Jahoda, C. A., Reynolds, A. J., Chaponnier, C., Forester, J. C. \& Gabbiani, G. Smooth muscle alpha-actin is a marker for hair follicle dermis in vivo and in vitro. J. Cell Sci. 99(Pt 3), 627-636 (1991).

41. Lawson, D., Harrison, M. \& Shapland, C. Fibroblast transgelin and smooth muscle SM22alpha are the same protein, the expression of which is downregulated in many cell lines. Cell Motil. Cytoskelet. 38, 250-257 (1997).

42. Grieskamp, T., Rudat, C., Ludtke, T. H., Norden, J. \& Kispert, A. Notch signaling regulates smooth muscle differentiation of epicardium-derived cells. Circ. Res. 108, 813-823 (2011).

43. Clements, M. P. et al. The wound microenvironment reprograms schwann cells to invasive mesenchymal-like cells to drive peripheral nerve regeneration. Neuron 96, 98-114.e117 (2017).

44. Ellinghaus, P. et al. Expression of pro-inflammatory genes in human endothelial cells: Comparison of rivaroxaban and dabigatran. Thromb. Res. 142, 44-51 (2016).

45. Rezza, A. et al. Signaling Networks among Stem Cell Precursors, TransitAmplifying Progenitors, and their Niche in Developing Hair Follicles. Cell Rep. 14, 3001-3018 (2016).

46. Rendl, M., Lewis, L. \& Fuchs, E. Molecular dissection of mesenchymalepithelial interactions in the hair follicle. PLoS Biol. 3, e331 (2005).

47. Driskell, R. R., Clavel, C., Rendl, M. \& Watt, F. M. Hair follicle dermal papilla cells at a glance. J. Cell Sci. 124, 1179-1182 (2011).
48. Rendl, M., Polak, L. \& Fuchs, E. BMP signaling in dermal papilla cells is required for their hair follicle-inductive properties. Genes Dev. 22, 543-557 (2008).

49. Xie, J. et al. Activating Smoothened mutations in sporadic basal-cell carcinoma. Nature 391, 90-92 (1998).

50. Wong, S. Y. \& Reiter, J. F. Wounding mobilizes hair follicle stem cells to form tumors. Proc. Natl Acad. Sci. USA 108, 4093-4098 (2011).

51. Youssef, K. K. et al. Identification of the cell lineage at the origin of basal cell carcinoma. Nat. Cell Biol. 12, 299-305 (2010).

52. Yang, S. H. et al. Pathological responses to oncogenic Hedgehog signaling in skin are dependent on canonical Wnt/beta3-catenin signaling. Nat. Genet. 40, 1130-1135 (2008)

53. Gay, D. et al. Fgf9 from dermal gammadelta $\mathrm{T}$ cells induces hair follicle neogenesis after wounding. Nat. Med. 19, 916-923 (2013).

54. Chen, D., Jarrell, A., Guo, C., Lang, R. \& Atit, R. Dermal beta-catenin activity in response to epidermal Wnt ligands is required for fibroblast proliferation and hair follicle initiation. Development 139, 1522-1533 (2012).

55. Wang, X. et al. Macrophages induce AKT/beta-catenin-dependent Lgr5(+) stem cell activation and hair follicle regeneration through TNF. Nat. Commun. 8, 14091 (2017).

56. Nelson, A. M. et al. dsRNA Released by Tissue Damage Activates TLR3 to Drive Skin Regeneration. Cell Stem Cell 17, 139-151 (2015).

57. Hamburg-Shields, E., DiNuoscio, G. J., Mullin, N. K., Lafyatis, R. \& Atit, R. P. Sustained beta-catenin activity in dermal fibroblasts promotes fibrosis by upregulating expression of extracellular matrix protein-coding genes. J. Pathol. 235, 686-697 (2015).

58. Rognoni, E. et al. Inhibition of beta-catenin signalling in dermal fibroblasts enhances hair follicle regeneration during wound healing. Development 143, 2522-2535 (2016).

59. Choi, Y. S. et al. Distinct functions for Wnt/beta-catenin in hair follicle stem cell proliferation and survival and interfollicular epidermal homeostasis. Cell Stem Cell 13, 720-733 (2013).

60. Ferrer-Vaquer, A. et al. A sensitive and bright single-cell resolution live imaging reporter of Wnt/ss-catenin signaling in the mouse. BMC Dev. Biol. 10, 121 (2010).

61. Gat, U., DasGupta, R., Degenstein, L. \& Fuchs, E. De novo hair follicle morphogenesis and hair tumors in mice expressing a truncated beta-catenin in skin. Cell 95, 605-614 (1998).

62. Oro, A. E. et al. Basal cell carcinomas in mice overexpressing sonic hedgehog. Science 276, 817-821 (1997).

63. Takeo, M., Lee, W. \& Ito, M. Wound healing and skin regeneration. Cold Spring Harb. Perspect. Med. 5, a023267 (2015).

64. Myung, P. S., Takeo, M., Ito, M. \& Atit, R. P. Epithelial Wnt ligand secretion is required for adult hair follicle growth and regeneration. J. Invest. Dermatol. 133, 31-41 (2013).

65. Harada, N. et al. Intestinal polyposis in mice with a dominant stable mutation of the beta-catenin gene. EMBO J. 18, 5931-5942 (1999).

66. Rabbani, P. et al. Coordinated activation of Wnt in epithelial and melanocyte stem cells initiates pigmented hair regeneration. Cell 145, 941-955 (2011).

67. Ito, M. et al. Stem cells in the hair follicle bulge contribute to wound repair but not to. Nat. Med. 11, 1351-1354 (2005).

68. Takeo, M. et al. EdnrB Governs Regenerative Response of Melanocyte Stem Cells by Crosstalk with Wnt Signaling. Cell Rep. 15, 1291-1302 (2016).

69. Huang da, W., Sherman, B. T. \& Lempicki, R. A. Systematic and integrative analysis of large gene lists using DAVID bioinformatics resources. Nat. Protoc. 4, 44-57 (2009).

70. Takeo, M. et al. Wnt activation in nail epithelium couples nail growth to digit regeneration. Nature 499, 228-232 (2013).

\section{Acknowledgements}

We thank the NYUMC Genome Technology Center for expert assistance with library preparation and Illumina sequencing (Karina Ray, Christian Marier, Paul Zappile, Yutong Zhang, Steven Shen (current address: University of Minnesota), Tenzin Lhakhang, and Adriana Heguy) We thank the NYUMC Experimental Pathology Research Laboratory for performing Masson's trichrome staining (Charly Zhao and Cynthia Loomis); These centers are partially supported by the Cancer Center Support Grant P30CA016087 at the Laura and Isaac Perlmutter Cancer Center. We thank the NYUMC DART Microscopy Lab for the assistance of microscopy analyses including TEM analysis (Kristen Dancel, Chris Petzold, Michael Cammer, Yan Deng, and Feng-Xia (Alice) Liang). We thank the NYUMC Rodent Genetic Engineering Laboratory for the rederivation of transgenic mice including $L S L$-Sh,$\beta$-catenin $f($ (ex3) and Axin2-CreER (Sang Yong Kim). We thank Denise Gay for her scientific inputs and valuable edits of the manuscript. Research reported in this publication was supported in part by the National Institute of Arthritis and Musculoskeletal and Skin Diseases, part of the National Institutes of Health under Award Numbers R01AR059768 (M.I.), R01AR066022 (M.I.), R01AR055309 (G.C.), and T32AR064184 (C.H.L.). The content is solely the responsibility of the authors and does not necessarily represent the official views of the National 
Institutes of Health. M.I is also supported by the Arnold and Mabel Beckman Foundation and the American Skin Association.

\section{Author contributions}

C.H.L. designed and performed most of the experiments, generated final figures, and wrote the manuscript. K.R., Q.S., designed and performed experiments/analyses and interpreted data. M.T., P.R., S.L., W.L., M.P., P.M., Y.Z, and G.C. collaborated on this project and shared data or mice that are essential to complete the project. N.W. in collaboration with J.E.C and D.H.W provided $L S L$-Shh mice. M.M.T. provided $\beta$-catenin $f($ ex3) mice. S.M. provided Axin2-CreER mice. M.I. designed the project, supervised experiments and wrote the manuscript.

\section{Additional information}

Supplementary Information accompanies this paper at https://doi.org/10.1038/s41467018-07142-9.

Competing interests: M.I. and G.C. are listed as inventors on a patent related to wounding-induced hair follicle neogenesis and owned by the University of Pennsylvania. M.I. and G.C. have equity in Follica, a start up company that has licensed this patent. All remaining authors declare no competing interests.
Reprints and permission information is available online at http://npg.nature.com/ reprintsandpermissions/

Publisher's note: Springer Nature remains neutral with regard to jurisdictional claims in published maps and institutional affiliations.

cc (i) Open Access This article is licensed under a Creative Commons Attribution 4.0 International License, which permits use, sharing, adaptation, distribution and reproduction in any medium or format, as long as you give appropriate credit to the original author(s) and the source, provide a link to the Creative Commons license, and indicate if changes were made. The images or other third party material in this article are included in the article's Creative Commons license, unless indicated otherwise in a credit line to the material. If material is not included in the article's Creative Commons license and your intended use is not permitted by statutory regulation or exceeds the permitted use, you will need to obtain permission directly from the copyright holder. To view a copy of this license, visit http://creativecommons.org/ licenses/by/4.0/.

(C) The Author(s) 2018 